\title{
Polynomial Invariants, Knot Homologies, and Higher Twist Numbers of Weaving Knots $W(3, n)$
}

\author{
Rama Mishra ${ }^{1}$ \\ Department of Mathematics \\ IISER Pune \\ Pune, India
}

\author{
Ross Staffeldt ${ }^{2}$ \\ Department of Mathematical Sciences \\ New Mexico State University \\ Las Cruces, NM 88003 USA
}

May 9, 2019

${ }^{1}$ We thank the office of the Dean of the College of Arts and Sciences, New Mexico State University, for arranging a visiting appointment.

${ }^{2}$ We thank the office of the Vice-President for Research, New Mexico State University, for arranging a visit to IISER-Pune to discuss implementation of an MOU between IISER-Pune and NMSU. 


\begin{abstract}
We investigate several conjectures in geometric topology by assembling computer data obtained by studying weaving knots, a doubly infinite family $W(p, n)$ of examples of hyperbolic knots. In particular, we compute some important polynomial knot invariants, as well as knot homologies, for the subclass $W(3, n)$ of this family. We use these knot invariants to conclude that all knots $W(3, n)$ are fibered knots and provide estimates for some geometric invariants of these knots. Finally, we study the asymptotics of the ranks of their Khovanov homology groups. Our investigations provide evidence for our conjecture that, asymptotically as $n$ grows large, the ranks of Khovanov homology groups of $W(3, n)$ are normally distributed.
\end{abstract}




\section{Introduction}

Distinguishing knots and links up to ambient isotopy is the central problem in knot theory. The recipe that a knot theorist uses is to compute some knot invariants and see if one of them can be of help. Classically one used the Alexander polynomial, which has a topological definition [16, p.160], to distinguish knots. Over the last 35 years tremendous progress has been made in the development of several new knot invariants, starting with the Jones polynomial and the HOMFLY-PT polynomial [6]. Recently even more sophisticated invariants such as Heegard-Floer homology groups [10] and Khovanov homology groups [1] have been added to the toolkit. These homology theories are called categorifications of polynomial invariants, because passage to an appropriate Euler characteristic retrieves a polynomial invariant. Though these invariants are well understood to a great extent, it is very difficult to compute them for a given knot whose crossing number is only moderately large. Moreover, some of these invariants yield a very large amount of numerical data, and the problem arises of parsing, or summarizing, the data in a reasonable way.

Since the Alexander polynomial can clearly be related to topology, it is natural to want to relate the new invariants to topological or geometric features of knots or links. In the 1980s William Thurston's seminal result [19, Corollary 2.5] that most knot complements have the structure of a hyperbolic manifold, combined with Mostow's rigidity theorem [19, Theorem 3.1] giving uniqueness of such structures, establishes a strong connection between hyperbolic geometry and knot theory, since knots are determined by their complements. Indeed, any geometric invariant of a knot complement, such as the hyperbolic volume, becomes a topological invariant of the knot. Thus, investigating if data derived from the new knot invariants is related to natural differential geometric invariants becomes another natural problem.

In this paper, we take up the family of weaving knots $W(p, n)$, where $p$ and $n$ are positive integers. We compute the signature for the general knot $W(p, n)$, and compute the polynomial knot invariants for the subfamily $W(3, n)$. We explore the two problems just mentioned using our computations. In particular, Dasbach and Lin [5] have provided some bounds on the hyperbolic volume of alternating knots in terms of coefficients of the Jones polynomial. They also define higher twist numbers for knots in terms of coefficients of the Jones polynomials and suggested that these may have some correlation with the hyperbolic volume of the knots. We investigate this idea for the $W(3, n)$ knots. As for parsing the enormous amount of numerical information yielded by our methods, we explore the approximation of normalized Khovanov homology by normal distributions from mathematical statistics. A preprint [12] developing this idea further is in preparation.

Let us pause for more explanation of our decision to focus on weaving knots. According to [3], these knots have recently attracted interest, because it was conjectured that their complements would have the largest hyperbolic volume for a fixed crossing number. Concerning the conjecture about the volume, we cite the following theorem.

Theorem 1 (Theorem 1.1, [3]). If $p \geq 3$ and $q \geq 7$, then

$$
v_{\text {oct }}(p-2) q\left(1-\frac{(2 \pi)^{2}}{q^{2}}\right)^{3 / 2} \leq \operatorname{vol}(W(p, q))<\left(v_{\text {oct }}(p-3)+4 v_{\text {tet }}\right) q \text {. }
$$

Here $v_{\text {oct }}$ and $v_{\text {tet }}$ denote the volumes of the ideal octahedron and ideal tetrahedron respectively. Champanerkar, Kofman, and Purcell call these bounds asymptotically sharp 
because their ratio approaches 1 , as $p$ and $q$ tend to infinity. Since the crossing number of $W(p, q)$ is known to be $(p-1) q$, the volume bounds in the theorem imply

$$
\lim _{p, q \rightarrow \infty} \frac{\operatorname{vol}(W(p, q))}{c(W(p, q))}=v_{\text {oct }} \approx 3.66
$$

Their study raises the general question of examining the asymptotic behaviour of other invariants of weaving knots. We also investigate the efficiency of the upper and lower bounds given in the theorem for weaving knots $W(3, n)$.

Turning to practical matters, here is the weaving knot $W(3,4)$.

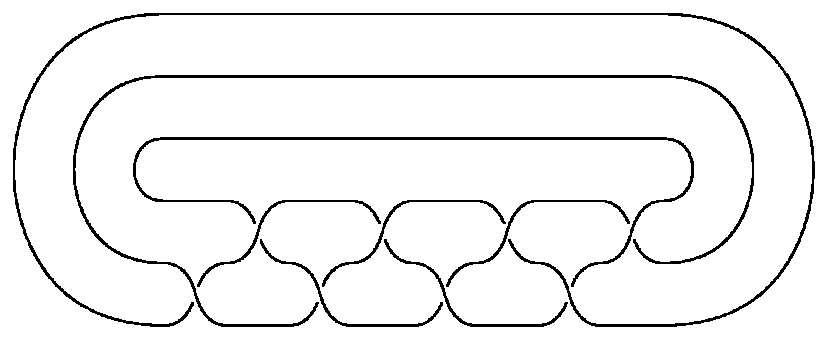

Enumerating strands $1, \ldots, p$ from the outside inward, our example is the closure of the braid $\left(\sigma_{1} \sigma_{2}^{-1}\right)^{4}$ on three strands. The weaving $\operatorname{knot} W(p, n)$ is obtained from the torus $\operatorname{knot} T(p, n)$ by making a standard diagram of the torus knot alternating. Symbolically, $T(p, n)$ is the closure of the braid $\left(\sigma_{1} \sigma_{2} \cdots \sigma_{p-1}\right)^{n}$, and $W(p, n)$ is the closure of the braid $\left(\sigma_{1} \sigma_{2}^{-1} \cdots \sigma_{p-1}^{ \pm 1}\right)^{n}$. Obviously, the parity of $p$ is important. If the greatest common divisor $\operatorname{gcd}(p, q)>1$, then $T(p, n)$ and $W(p, n)$ are both links with $\operatorname{gcd}(p, n)$ components. In general we are interested only in the cases when $W(p, n)$ is an actual knot. The first invariant that we compute for $W(p, n)$ is the signature $\sigma(W(p, n))$ using a combinatorial method useful for alternating knot diagrams discussed in [9]. We then focus on the knots $W(3, n)$ which are closures of 3-strand braids.

From early in the development of Khovanov homology, computer experimentation has played a role in advancing the theory. For example, [1] provided Mathematica routines to compute Khovanov homology of knots of up to 10 crossings and provided tables of Betti numbers. Based on the computations, he makes a number of observations and conjectures about the structure of Khovanov homology. Subsequently, 8] recorded some observations about patterns in Khovanov homology and a remarkable relationship between the volume of a knot complement and the total rank of the knot's Khovanov homology. Some of the conjectures on patterns are proved in [9], on which our results depend. In this paper we start a study of the asymptotic behavior of Khovanov homology of weaving knots. With the assistance of the computer algebra system Maple, we provide data on the Khovanov homology of weaving knots $W(3, n)$ with up to 652 crossings. The Khovanov homology groups are truly enormous, so we approximate the distribution of dimensions using probability density functions. We find that normal distributions fit the data surprisingly well.

In more detail, this paper is organized as follows. In section 2 , we discuss the generalities of weave knots and compute the signature $\sigma(W(p, n))$. We also make observation on Rasmussen's invariant [15] for these knots. In section 3] we prepare to follow the development of polynomial invariants in [6], starting from representations of braid groups into Hecke algebras. For weaving knots $W(3, n)$, which are naturally represented as the closures of braids on 
three strands, we develop recursive formulas for their representations in the Hecke algebras. We note that the survey [2] collects a number of facts and tools to facilitate computations of invariants of knots and links that are the closures of 3-strand braids, as well as classifying the prime knots that are closure of 3 -strand braids but not of 2 -strand braids. We would also like to point out that [18] studies the Jones polynomials of knots that are the closures of general 3-strand braids, but the method is based on the skein relation satisfied by the Jones polynomial. Our formulas are used not only in computer calculations of the polynomial invariants we need, but also in the development of information about particular coefficients of these polynomials.

Section 4 builds on the recursion formulas to develop information about the Alexander polynomial $\Delta_{W(3, n)}(t)$ and Jones polynomial $V_{W(3, n)}(t)$. As an application we exploit the relation between the Heegard-Floer homology associated to an alternating knot and its Alexander polynomial given in [13, Theorem 1.3], as well as properties of Heegard-Floer homology, to prove that the complements of the knots $W(3, n)$ are fibered over $S^{1}$.

In section 5 we investigate for knots $W(3, n)$ the higher twist numbers defined by Dasbach and Lin in [5] in terms of the Jones polynomial. They ask if there is a correlation between the values of the higher twist numbers and the hyperbolic volume of the knot complements. As new results we have formulas for the second and third twist numbers of the knots $W(3, n)$, as well as conjectures for even higher twist numbers. We believe that improved lower bounds on the volume of knots $W(3, n)$ can be derived from the higher twist numbers, displaying results of computer experiments to support this idea. We also exhibit plots of higher twist numbers against volume that support the idea that values of higher twist numbers are correlated with volume.

In section [6 we explain how to obtain the two-variable Poincaré polynomial for Khovanov homology with rational coefficients, and we present the results of calculations in a few relatively small examples. Using recent results of Shumakovitch [17] that explain how, for alternating knots, the rational Khovanov homology determines the integral Khovanov homology, we provide a display of the integral Khovanov homology of the knot $W(3,4)$. Concerning rational Khovanov homology, we observe that the distributions of dimensions in Khovanov homology resemble normal distributions. We explore this further in section 8 , where we present tables displaying summaries of calculations for weaving knots $W(3, n)$ for selected values of $n$ satisfying $\operatorname{gcd}(3, n)=1$ and ranging up to $n=326$. The standard deviation $\sigma$ of the normal distribution we attach to the Khovanov homology of a weaving knot is a significant parameter. The geometric significance of this number is an open question.

In section 9 we display expressions for a few polynomials arising from the Hecke algebra representations of braid representations of $W(3, n)$, as they are used in section 5, and values of the Jones polynomial, Alexander polynomial and HOMFLY-PT polynomial for knots $W(3,4), W(3,5), W(3,10)$ and $W(3,11)$. Finally, in section 10 we provide some information about the computer experiments we have performed with the knots $W(3, n)$ and how the experimental results influenced the formulations of propositions and theorems.

Finally, we thank several colleagues, especially Dr. Joan Birman, for advice and suggestions concerning the exposition. 


\section{Generalities on Weaving knots}

We have already mentioned that weaving knots are alternating by definition. Various facts about alternating knots facilitate our calculations of the Khovanov homology of weaving knots $W(3, n)$. For example, we appeal first to the following theorem of Lee.

Theorem 2.1 (Theorem 1.2, 9]). For any alternating knot L the Khovanov invariants $\mathcal{H}^{i, j}(L)$ are supported in two lines

$$
j=2 i-\sigma(L) \pm 1 \text {. }
$$

We will see that this result also has several practical implications. For example, to obtain a vanishing result for a particular alternating knot, it suffices to compute the signature. Likewise, in connection with Heegard-Floer homology for $W(p, n)$, [13, Theorem 1.3] essentially says that Heegard-Floer homology for an alternating knot is completely determined by the coefficients of its Alexander polynomial and the signature.

Thus, it is important to compute the signature. Indeed, it turns out that there is a combinatorial formula for the signature of oriented non-split alternating links. To state the formula requires the following terminology.

Definition 2.2. For a link diagram $D$ let $c(D)$ be the number of crossings of $D$, let $x(D)$ be number of negative crossings, and let $y(D)$ be the number of positive crossings. For an oriented link diagram, let $o(D)$ be the number of components of $D(\emptyset)$, the diagram obtained by $A$-smoothing every crossing.
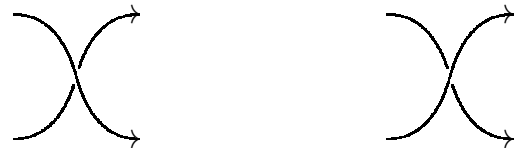

Figure 1: Positive and negative crossings
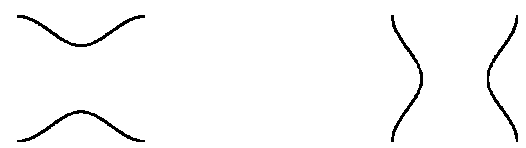

Figure 2: $A$-smoothing a positive, resp., negative, crossing

In words, $A$-regions in a neighborhood of a crossing are the regions swept out as the upper strand sweeps counter-clockwise toward the lower strand. An $A$-smoothing removes the crossing to connect these regions. With these definitions, we may cite the following proposition.

Proposition 2.3 (Proposition 3.11, 9]). For an oriented non-split alternating link $L$ and a reduced alternating diagram $D$ of $L, \sigma(L)=o(D)-y(D)-1$.

We now use this result to compute the signatures of weaving knots. For a knot or link $W(m, n)$ drawn in the usual way, the number of crossings $c(D)=(m-1) n$. In particular, for $W(2 k+1, n), c(W(2 k+1, n))=2 k n$; for $W(2 k, n), c(W(2 k, n))=(2 k-1) n$. Evaluating the other quantities in definition 2.2 , we calculate the signatures of weaving knots.

Proposition 2.4. For a weaving knot $W(2 k+1, n), \sigma(W(2 k+1, n))=0$, and for $W(2 k, n)$, $\sigma(W(2 k, n))=-n+1$. 
Corollary 2.5. For a weaving knot $W(2 k+1, n)$, the Rasmussen s-invariant is zero. For a weaving knot $W(2 k, n)$, the Rasmussen s-invariant is $-n+1$.

Proof. For alternating knots, it is known that the $s$-invariant coincides with the signature [15, Theorems 1-4].

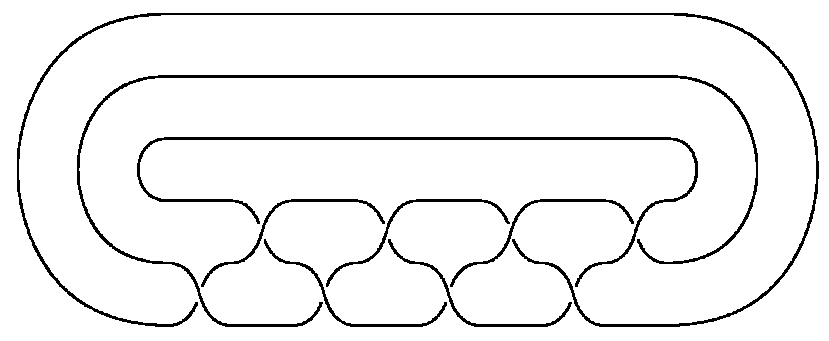

Figure 3: The weaving knot $W(3,4)$

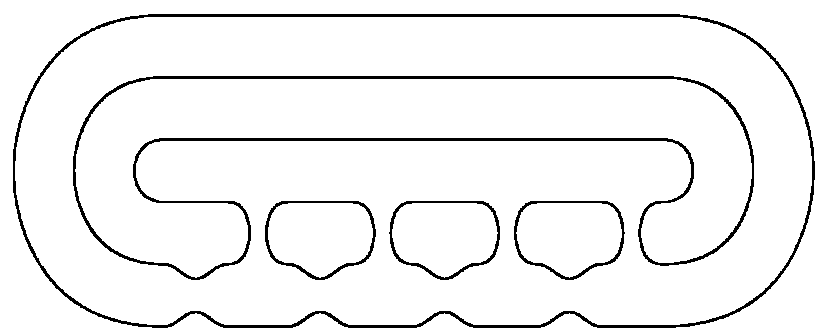

Figure 4: The $A$-smoothing of $W(3,4)$

Proof of Proposition 2.4. Consider first the example $W(3, n)$, illustrated by figures 3 and 4 for $W(3,4)$. After $A$-smoothing the diagram, the outer ring of crossings produces a circle bounding the rest of the smoothed diagram. On the inner ring of crossings the $A$-smoothings produce $n$ circles in a cyclic arrangement. Therefore $o(W(3, n))=1+n$. The outer ring of crossings consists of positive crossings and the inner ring of crossings consists of negative crossings, so $x(D)=y(D)=n$. Applying the formula of proposition 2.3, we obtain the result $\sigma(W(3, n))=0$.

For the general case $W(2 k+1, n)$, we have the following considerations. The crossings are organized into $2 k$ rings. Reading from the outside toward the center, we have first a ring of positive crossings, then a ring of negative crossings, and so on, alternating positive and negative. Thus, $y(D)=k n$. Considering the $A$-smoothing of the diagram of $W(2 k+1, n)$, as in the special case, a bounding circle appears from the smoothing of the outer ring. A chain of $n$ disjoint smaller circles appears inside the second ring. No circles appear in the third ring, nor in any odd-numbered ring thereafter. On the other hand, chains of $n$ disjoint smaller circles appear in each even-numbered ring. Since there are $k$ even-numbered rings, we have $o(D)=1+k n$. Applying the formula of proposition 2.3

$$
\sigma(W(2 k+1, n))=o(D)-y(D)-1=(1+k n)-k n-1=0 .
$$




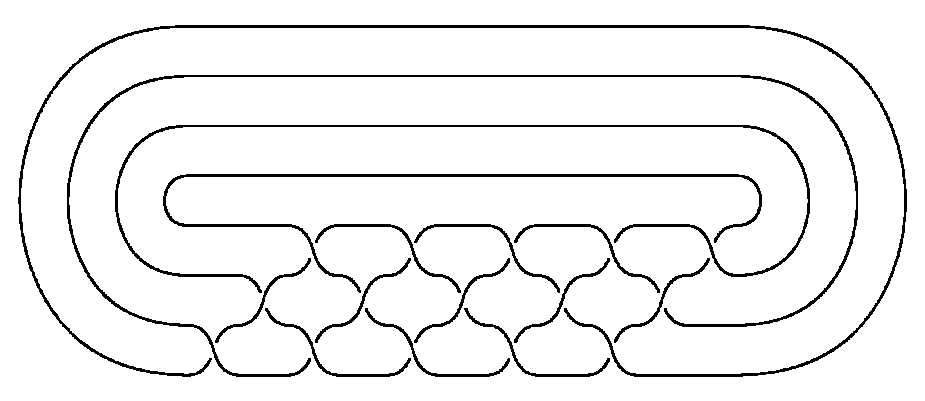

Figure 5: The weaving knot $W(4,5)$

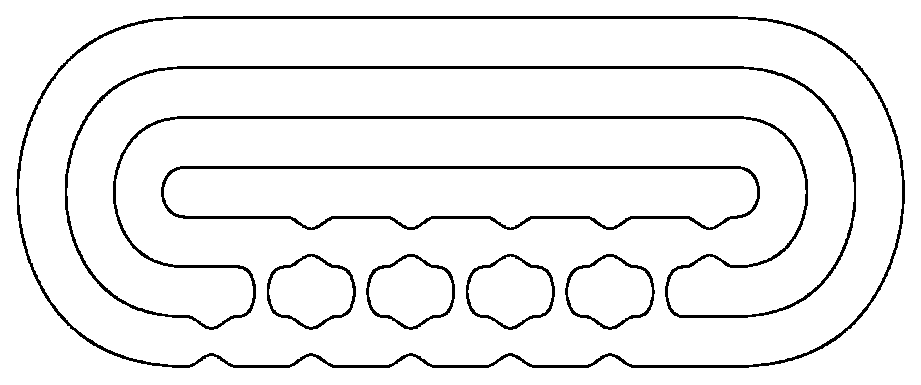

Figure 6: The $A$-smoothing of $W(4,5)$

For the case $W(2 k, n)$, we show $W(4,5)$ in figures 5 and 6 as an example. Our standard diagram may be organized into $2 k-1$ rings of crossings. In each ring there are $n$ crossings, so the total number of crossings is $c(D)=(2 k-1) n$. In our standard representation, there is an outer ring of $n$ positive crossings, next a ring of $n$ negative crossings, alternating until we end with an innermost ring of $n$ positive crossings. There are thus $k$ rings of $n$ positive crossings and $k-1$ rings of $n$ negative crossings. Therefore, $y(D)=k n$ and $x(D)=(k-1) n$. Considering the $A$-smoothing of the diagram, a bounding circle appears from the smoothing of the outer ring. As before, a chain of $n$ disjoint smaller circles appears inside the second ring and in each successive even-numbered ring. As previously noted, there are $k-1$ of these rings. No circles appear in odd-numbered rings, until we reach the last ring, where an inner bounding circle appears. Thus, $o(D)=1+(k-1) n+1=(k-1) n+2$. Consequently,

$$
\sigma(W(2 k, n))=o(D)-y(D)-1=((k-1) n+2)-k n-1=-n+1 .
$$

Theorem 2.6. For a weaving knot $W(2 k+1, n)$ the non-vanishing Khovanov homology $\mathcal{H}^{i, j}(W(2 k+1, n))$ lies on the lines

$$
j=2 i \pm 1 \text {. }
$$

For a weaving knot $W(2 k, n)$ the non-vanishing Khovanov homology $\mathcal{H}^{i, j}(W(2 k, n))$ lies on the lines

$$
j=2 i+n-1 \pm 1
$$

Proof. Substitute the calculations made in proposition 2.4 into the formula of theorem 2.1. 


\section{Recursion in the Hecke algebra}

We review briefly the definition of the Hecke algebra $H_{N+1}$ on generators 1 and $T_{1}$ through $T_{N}$, and we define the representation of the braid group $B_{3}$ on three strands in $H_{3}$. Theorem 3.2 sets up recursion relations for the coefficients in the expansion of the image in $\mathrm{H}_{3}$ of the braid $\left(\sigma_{1} \sigma_{2}^{-1}\right)^{n}$, whose closure is the weaving knot $W(3, n)$. These coefficients are polynomials in a parameter $q$, which is built into the definition 3.1 of the Hecke algebra. The recursion relations are essential for automating the calculation of the Jones polynomial for the knots $W(3, n)$. Proposition 3.4 uses the relations developed in theorem 3.2 to prove a vanishing result for one of the coefficients. Being able to ignore one of the coefficients speeds up the computations slightly. Proposition 3.5 evaluates the constant terms of the families of polynomials, proposition 3.6 evaluates the degree one coefficients, and theorem 3.7 proves certain identities satisfied by the polynomials. These identities imply symmetry properties of the coefficients and enable calculation of the degrees of the polynomials in corollary 3.8 .

Definition 3.1. Working over the ground field $K$ containing an element $q \neq 0$, the Hecke algebra $H_{N+1}$ is the associative algebra with 1 on generators $T_{1}, \ldots, T_{N}$ satisfying these relations.

$$
\begin{aligned}
T_{i} T_{j} & =T_{j} T_{i}, \quad \text { whenever }|i-j| \geq 2, \\
T_{i} T_{i+1} T_{i} & =T_{i+1} T_{i} T_{i+1}, \quad \text { for } 1 \leq i \leq N-1,
\end{aligned}
$$

and, finally,

$$
T_{i}^{2}=(q-1) T_{i}+q, \quad \text { for all } i
$$

It is well-known [6] that $(N+1)$ ! is the dimension of $H_{N+1}$ over $K$.

Recasting the relation $T_{i}^{2}=(q-1) T_{i}+q$ in the form $q^{-1}\left(T_{i}-(q-1)\right) \cdot T_{i}=1$ shows that $T_{i}$ is invertible in $H_{N+1}$ with $T_{i}^{-1}=q^{-1}\left(T_{i}-(q-1)\right)$. Consequently, the specification $\rho\left(\sigma_{i}\right)=T_{i}$, combined with relations (3.1) and (3.2), defines a homomorphism $\rho: B_{N+1} \rightarrow H_{N+1}$ from $B_{N+1}$, the group of braids on $N+1$ strands, into the multiplicative monoid of $H_{N+1}$.

For work in $H_{3}$, choose the ordered basis $\left\{1, T_{1}, T_{2}, T_{1} T_{2}, T_{2} T_{1}, T_{1} T_{2} T_{1}\right\}$. The word in the Hecke algebra corresponding to the knot $W(3, n)$ is formally

$$
\rho\left(\left(T_{1} T_{2}^{-1}\right)^{n}\right)=q^{-n}\left(C_{n, 0}+C_{n, 1} \cdot T_{1}+C_{n, 2} \cdot T_{2}+C_{n, 12} \cdot T_{1} T_{2}+C_{n, 21} \cdot T_{2} T_{1}+C_{n, 121} \cdot T_{1} T_{2} T_{1}\right),
$$

where the coefficients $C_{n, *}=C_{n, *}(q)$ of the monomials in $T_{1}$ and $T_{2}$ are polynomials in $q$. For $n=1$,

$$
\rho\left(\sigma_{1} \sigma_{2}^{-1}\right)=T_{1} T_{2}^{-1}=q^{-1} \cdot\left(T_{1}\left(-(q-1)+T_{2}\right)\right)=q^{-1}\left(-(q-1) \cdot T_{1}+T_{1} T_{2}\right),
$$

so we have initial values

$$
C_{1,0}(q)=0, C_{1,1}(q)=-(q-1), C_{1,2}(q)=0, C_{1,12}(q)=1, C_{1,21}(q)=0, \text { and } C_{1,121}(q)=0
$$


Theorem 3.2. These polynomials satisfy the following recursion relations.

$$
\begin{aligned}
C_{n, 0}(q) & =q^{2} \cdot C_{n-1,21}(q)-q(q-1) \cdot C_{n-1,1}(q) \\
C_{n, 1}(q) & =-(q-1)^{2} \cdot C_{n-1,1}(q)-(q-1) \cdot C_{n-1,0}(q)+q^{2} \cdot C_{n-1,121}(q) \\
C_{n, 2}(q) & =q \cdot C_{n-1,1}(q) \\
C_{n, 12}(q)= & (q-1) \cdot C_{n-1,1}(q)+C_{n-1,0}(q) \\
C_{n, 21}(q)= & -(q-1) \cdot C_{(n-1), 2}(q)+q \cdot C_{n-1,12}(q) \\
& \quad-(q-1)^{2} \cdot C_{n-1,21}(q)+q(q-1) \cdot C_{n-1,121}(q) \\
C_{n, 121}(q)= & C_{n-1,2}(q)+(q-1) \cdot C_{n-1,21}(q)
\end{aligned}
$$

Proof. We have

$$
\begin{aligned}
& \rho\left(T_{1} T_{2}^{-1}\right)^{n}=\rho\left(T_{1} T_{2}^{-1}\right)^{n-1} \cdot \rho\left(T_{1} T_{2}^{-1}\right) \\
& =q^{-n+1}\left(C_{n-1,0}+C_{n-1,1} \cdot T_{1}+C_{n-1,2} \cdot T_{2}+C_{n-1,12} \cdot T_{1} T_{2}+C_{n-1,21} \cdot T_{2} T_{1}+C_{n-1,121} \cdot T_{1} T_{2} T_{1}\right) \\
& \cdot q^{-1}\left(-(q-1) \cdot T_{1}+T_{1} T_{2}\right) \\
& =q^{-n}\left(-(q-1) C_{n-1,0} \cdot T_{1}-(q-1) C_{n-1,1} \cdot T_{1}^{2}-(q-1) C_{n-1,2} \cdot T_{2} T_{1}\right. \\
& -(q-1) C_{n-1,12} \cdot T_{1} T_{2} T_{1}-(q-1) C_{n-1,21} \cdot T_{2} T_{1}^{2}-(q-1) C_{n-1,121} \cdot T_{1} T_{2} T_{1}^{2} \\
& +C_{n-1,0} \cdot T_{1} T_{2}+C_{n-1,1} \cdot T_{1}^{2} T_{2}+C_{n-1,2} \cdot T_{2} T_{1} T_{2} \\
& \left.+C_{n-1,12} \cdot T_{1} T_{2} T_{1} T_{2}+C_{n-1,21} \cdot T_{2} T_{1}^{2} T_{2}+C_{n-1,121} \cdot T_{1} T_{2} T_{1}^{2} T_{2}\right) \\
& =q^{-n}\left(\left(-(q-1) C_{n-1,0} \cdot T_{1}-(q-1) C_{n-1,2} \cdot T_{2} T_{1}-(q-1) C_{n-1,12} \cdot T_{1} T_{2} T_{1}+C_{n-1,0} \cdot T_{1} T_{2}\right)\right. \\
& +\left\{-(q-1) C_{n-1,1} \cdot T_{1}^{2}-(q-1) C_{n-1,21} \cdot T_{2} T_{1}^{2}-(q-1) C_{n-1,121} \cdot T_{1} T_{2} T_{1}^{2}+C_{n-1,1} \cdot T_{1}^{2} T_{2}\right. \\
& \left.\left.+C_{n-1,2} \cdot T_{2} T_{1} T_{2}+C_{n-1,12} \cdot T_{1} T_{2} T_{1} T_{2}+C_{n-1,21} \cdot T_{2} T_{1}^{2} T_{2}+C_{n-1,121} \cdot T_{1} T_{2} T_{1}^{2} T_{2}\right\}\right)
\end{aligned}
$$

after collecting powers of $q$ and expanding. In the last grouping, the first four terms inside the parentheses ( ) involve only elements of the preferred basis; the second eight terms in the pair of braces \{\} all require further expansion, as follows.

$$
\begin{aligned}
-(q-1) C_{n-1,1} \cdot T_{1}^{2} & =-(q-1) C_{n-1,1} \cdot\left((q-1) T_{1}+q\right) \\
& =-(q-1)^{2} C_{n-1,1} \cdot T_{1}-q(q-1) C_{n-1,1} \\
-(q-1) C_{n-1,21} \cdot T_{2} T_{1}^{2} & =-(q-1) C_{n-1,21} \cdot T_{2}\left((q-1) T_{1}+q\right) \\
& =-(q-1)^{2} C_{n-1,21} \cdot T_{2} T_{1}-q(q-1) C_{n-1,21} \cdot T_{2} \\
-(q-1) C_{n-1,121} \cdot T_{1} T_{2} T_{1}^{2} & =-(q-1) C_{n-1,121} \cdot T_{1} T_{2}\left((q-1) T_{1}+q\right) \\
& =-(q-1)^{2} C_{n-1,121} \cdot T_{1} T_{2} T_{1}-q(q-1) C_{n-1,121} \cdot T_{1} T_{2} \\
C_{n-1,1} \cdot T_{1}^{2} T_{2} & =C_{n-1,1} \cdot\left((q-1) T_{1}+q\right) T_{2} \\
& =(q-1) C_{n-1,1} \cdot T_{1} T_{2}+q C_{n-1,1} \cdot T_{2} \\
C_{n-1,2} \cdot T_{2} T_{1} T_{2} & =C_{n-1,2} \cdot T_{1} T_{2} T_{1}
\end{aligned}
$$




$$
\begin{aligned}
C_{n-1,12} \cdot T_{1} T_{2} T_{1} T_{2} & =C_{n-1,12} \cdot T_{1}^{2} T_{2} T_{1}=C_{n-1,12}\left((q-1) T_{1}+q\right) T_{2} T_{1} \\
& =(q-1) C_{n-1,12} \cdot T_{1} T_{2} T_{1}+q C_{n-1,12} \cdot T_{2} T_{1} \\
C_{n-1,21} \cdot T_{2} T_{1}^{2} T_{2}= & C_{n-1,21} \cdot T_{2}\left((q-1) T_{1}+q\right) T_{2} \\
= & (q-1) C_{n-1,21} \cdot T_{2} T_{1} T_{2}+q C_{n-1,21} \cdot T_{2}^{2} \\
= & (q-1) C_{n-1,21} \cdot T_{1} T_{2} T_{1}+q C_{n-1,21} \cdot\left((q-1) T_{2}+q\right) \\
= & \left.(q-1) C_{n-1,21} \cdot T_{1} T_{2} T_{1}+q(q-1) C_{n-1,21} \cdot T_{2}+q^{2} C_{n-1,21}\right) \\
C_{n-1,121} \cdot T_{1} T_{2} T_{1}^{2} T_{2}= & C_{n-1,121} \cdot T_{1} T_{2}\left((q-1) T_{1}+q\right) T_{2} \\
= & (q-1) C_{n-1,121} \cdot T_{1} T_{2} T_{1} T_{2}+q C_{n-1,121} \cdot T_{1} T_{2}^{2} \\
= & (q-1) C_{n-1,121} \cdot T_{1}^{2} T_{2} T_{1}+q C_{n-1,121} \cdot T_{1}\left((q-1) T_{2}+q\right) \\
= & (q-1) C_{n-1,121} \cdot\left((q-1) T_{1}+q\right) T_{2} T_{1} \\
& \quad+q C_{n-1,121} \cdot T_{1}\left((q-1) T_{2}+q\right) \\
= & (q-1)^{2} C_{n-1,121} \cdot T_{1} T_{2} T_{1}+q(q-1) C_{n-1,121} \cdot T_{2} T_{1} \\
& \quad+q(q-1) C_{n-1,121} \cdot T_{1} T_{2}+q^{2} C_{n-1,121} \cdot T_{1}
\end{aligned}
$$

Collecting the constant terms from (3.13) and (3.19), we get

$$
C_{n, 0}=-q(q-1) C_{n-1,1}+q^{2} C_{n-1,21}
$$

Collecting coefficients of $T_{1}$ from (3.12), (3.13), (3.20), we get

$$
C_{n, 1}=-(q-1) C_{n-1,0}-(q-1)^{2} C_{n-1,1}+q^{2} C_{n-1,121}
$$

Collecting coefficients of $T_{2}$ from (3.14), (3.16), and (3.19), we get

$$
C_{n, 2}=-q(q-1) C_{n-1,21}+q C_{n-1,1}+q(q-1) C_{n-1,21}=q C_{n-1,1} .
$$

Collecting coefficients of $T_{1} T_{2}$ from (3.12), (3.15), (3.16), and (3.20), we get

$$
C_{n, 12}=C_{n-1,0}-q(q-1) C_{n-1,121}+(q-1) C_{n-1,1}+q(q-1) C_{n-1,121}=C_{n-1,0}+(q-1) C_{n-1,1}
$$

Collecting coefficients of $T_{2} T_{1}$ from (3.12), (3.14), (3.18), and (3.20), we get

$$
C_{n, 21}=-(q-1) C_{n-1,2}-(q-1)^{2} C_{n-1,21}+q C_{n-1,12}+q(q-1) C_{n-1,121} .
$$

Collecting coefficients of $T_{1} T_{2} T_{1}$ from (3.12), (3.15), (3.17), (3.18), (3.19), and (3.20), we get

$$
\begin{aligned}
C_{n, 121}= & -(q-1) C_{n-1,12}-(q-1)^{2} C_{n-1,121}+C_{n-1,2} \\
& \quad+(q-1) C_{n-1,12}+(q-1) C_{n-1,21}+(q-1)^{2} C_{n-1,121} \\
= & C_{n-1,2}+(q-1) C_{n-1,21}
\end{aligned}
$$

Up to simple rearrangements and expansion of notation, these are formulas (3.6) through (3.11). 
Example 3.3. Applying the recursion formulas just proved to the table of initial polynomials, or by computing $\rho\left(\left(\sigma_{1} \sigma_{2}^{-1}\right)^{2}\right)$ directly from the definitions, we find

$$
\begin{aligned}
C_{2,0}(q) & =q^{2} \cdot C_{1,21}(q)-q(q-1) \cdot C_{1,1}(q)=q(q-1)^{2}, \\
C_{2,1}(q) & =-(q-1)^{2} \cdot C_{1,1}(q)-(q-1) \cdot C_{1,0}(q)=(q-1)^{3}, \\
C_{2,2}(q) & =q \cdot C_{1,1}(q)=-q(q-1) \\
C_{2,12}(q) & =(q-1) \cdot C_{1,1}(q)+C_{1,0}(q)=-(q-1)^{2}, \\
C_{2,21}(q) & =-(q-1) \cdot C_{1,2}(q)+q \cdot C_{1,12}(q)-(q-1)^{2} \cdot C_{1,21}(q)=q, \\
C_{2,121}(q) & =0 .
\end{aligned}
$$

As a first application, we have the following vanishing result.

Proposition 3.4. For all $n, C_{n, 121}(q)=0$.

Proof. For $n \geq 1$, we claim $C_{n+1,121}(q)=0$. Make the inductive assumption that $C_{k, 121}(q)=$ 0 for $1 \leq k \leq n$. Apply (3.11), (3.10), and the inductive hypothesis to write

$$
\begin{aligned}
& C_{n+1,121}(q)=C_{n, 2}(q)+(q-1) \cdot C_{n, 21}(q) \\
& \begin{aligned}
=C_{n, 2}(q) \\
\quad+(q-1)\left(-(q-1) \cdot C_{n-1,2}(q)+q \cdot C_{n-1,12}(q)-(q-1)^{2} \cdot C_{n-1,21}(q)+q(q-1) \cdot C_{n-1,121}\right) \\
\quad=C_{n, 2}(q)+(q-1)\left(-(q-1) \cdot C_{n-1,2}(q)+q \cdot C_{n-1,12}(q)-(q-1)^{2} \cdot C_{n-1,21}(q)\right) .
\end{aligned}
\end{aligned}
$$

Using (3.8) to replace the first term $C_{n, 2}(q)$ and (3.9) to replace the third term factor $C_{n-1,12}(q)$ on the right,

$$
\begin{aligned}
& C_{n+1,121}(q)= \cdot C_{n-1,1}(q)-(q-1)^{2} C_{n-1,2}(q)+q(q-1)\left((q-1) C_{n-2,1}(q)+C_{n-2,0}(q)\right) \\
&-(q-1)^{3} C_{n, 21}(q) \\
&= \cdot C_{n-1,1}(q)-(q-1)^{2} C_{n-1,2}(q)+(q-1)^{2}\left(q C_{n-2,1}(q)\right)+q(q-1) C_{n-2,0}(q) \\
&-(q-1)^{3} C_{n, 21}(q) \\
&= \cdot C_{n-1,1}(q)-(q-1)^{2} C_{n-1,2}(q)+(q-1)^{2} C_{n-1,2}(q)+q(q-1) C_{n-2,0}(q) \\
& \quad-(q-1)^{3} C_{n, 21}(q),
\end{aligned}
$$

where we use (3.8) in reverse to rewrite the term $q C_{n-2,1}(q)$. Making the obvious cancellation,

$$
\begin{aligned}
C_{n+1,121} & =q \cdot C_{n-1,1}(q)+q(q-1) \cdot C_{n-2,0}(q)-(q-1)^{3} \cdot C_{n, 21}(q) \\
& =q\left(C_{n-1,1}+(q-1) C_{n-2,0}\right)-(q-1)^{3} \cdot C_{n-1,21} \\
& =q\left(\left(-(q-1)^{2} \cdot C_{n-2,1}-(q-1) \cdot C_{n-2,0}\right)+(q-1) \cdot C_{n-2,0}\right)-(q-1)^{3} \cdot C_{n-1,21},
\end{aligned}
$$

since

$$
\begin{aligned}
C_{n-1,1}(q) & =-(q-1)^{2} \cdot C_{n-2,1}(q)-(q-1) \cdot C_{n-2,0}(q)+q^{2} \cdot C_{n-2,121}(q) \\
& =-(q-1)^{2} \cdot C_{n-2,1}(q)-(q-1) \cdot C_{n-2,0}(q)
\end{aligned}
$$


by (3.7) and the inductive hypothesis. Therefore,

$$
\begin{aligned}
C_{n+1,121}(q) & =-q(q-1)^{2} \cdot C_{n-2,1}(q)-(q-1)^{3} \cdot C_{n-1,21}(q) \\
& =-(q-1)^{2} \cdot C_{n-1,2}(q)-(q-1)^{3} \cdot C_{n-1,21}(q),
\end{aligned}
$$

using (3.8) in the form $C_{n-1,2}(q)=q \cdot C_{n-2,1}(q)$,

$$
\begin{aligned}
& =-(q-1)^{2}\left(C_{n-1,2}(q)-(q-1) \cdot C_{n-1,21}(q)\right) \\
& =-(q-1)^{2} \cdot C_{n, 121}(q)=0,
\end{aligned}
$$

using (3.11) and the inductive hypothesis.

Proposition 3.5. For $n \geq 1$, the degree 0 terms in the non-vanishing polynomials $C_{n,-}$ are as follows:

$$
\begin{gathered}
C_{n, 0}(0)=c_{n, 0,0}=0, C_{n, 1}(0)=c_{n, 1,0}=(-1)^{n-1}, C_{n, 2}(0)=c_{n, 2,0}=0, \\
C_{n, 12}(0)=c_{n, 12,0}=(-1)^{n-1}, C_{n, 21}(0)=c_{n, 21,0}=0 .
\end{gathered}
$$

Since proposition 3.4 proves that $C_{n, 121}(q)$ is identically zero, it does not appear in the list just given or anywhere in the later parts of this paper.

Proof. Examinination of the formulas for the polynomials $C_{1,-}(q)$ given in equations (3.5) and for $C_{2,-}(q)$ given in example 3.3 starts the inductive proof. Substituting $q=0$ in the recursive formulas (3.6) and (3.8) immediately yields $C_{n, 0}(0)=0$ and $C_{n, 2}(0)=0$. Substituting $q=0$ into formula (3.7) yields

$$
c_{n, 1,0}=C_{n, 1}(0)=-(0-1)^{2} \cdot C_{n-1,1}(0)-(0-1) \cdot C_{n-1,0}(0)=-(-1)^{n-2}+0=(-1)^{n-1},
$$

by the inductive hypothesis. Similarly, substituting $q=0$ into formula (3.9) yields

$$
c_{n, 12,0}=C_{n, 12}(0)=(0-1) \cdot C_{n-1,1}(0)+C_{n-1,0}(0)=(-1) \cdot(-1)^{n-2}+0=(-1)^{n-1} .
$$

Finally, substituting $q=0$ into formula (3.10) yields

$$
\begin{aligned}
c_{n, 21,0}=C_{n, 21}(0)=-(0-1) \cdot C_{n-1,2}(0)+0 \cdot C_{n-1,12}(0)-(0-1)^{2} \cdot C_{n-1,21}(0) & \\
=1 \cdot 0+0 \cdot(-1)^{n-2}-1 \cdot 0 & =0 .
\end{aligned}
$$

Proposition 3.6. For $n \geq 2$ the degree one coefficients in the non-vanishing polynomials $C_{n,-}(q)$ are as follows:

$$
\begin{gathered}
c_{n, 0,1}=(-1)^{n}, \quad c_{n, 1,1}=(-1)^{n}(n+1), \quad c_{n, 2,1}=(-1)^{n}, \\
c_{n, 12,1}=(-1)^{n} n, \quad c_{n, 21,1}=(-1)^{n} .
\end{gathered}
$$

Proof. These are all handled in the same manner. Namely, differentiate the recursive relations for each successive polynomial, substitute $q=0$, and use the values from proposition 3.5 as appropriate. Concerning $C_{n, 0}(q)$, differentiate (3.6) with respect to $q$, obtaining

$$
C_{n, 0}^{\prime}(q)=2 q \cdot C_{n-1,21}(q)+q^{2} C_{n-1,21}^{\prime}(q)-(2 q-1) \cdot C_{n-1,1}(q)-q(q-1) \cdot C_{n-1,1}^{\prime}(q),
$$


whence

$$
c_{n, 0,1}=C_{n, 0}^{\prime}(0)=-(-1) \cdot C_{n-1,1}(0)=(-1)^{n-2}=(-1)^{n} .
$$

Concerning $C_{n, 1}(q)$, differentiate (3.7) with respect to $q$, obtaining

$$
C_{n, 1}^{\prime}(q)=-2(q-1) \cdot C_{n-1,1}(q)-(q-1)^{2} \cdot C_{n-1,1}^{\prime}(q)-C_{n-1,0}(q)-(q-1) \cdot C_{n-1,0}^{\prime}(q),
$$

whence

$$
\begin{aligned}
c_{n, 1,1}=C_{n, 1}^{\prime}(0)=2 \cdot C_{n-1,1}(0)-C_{n-1,1}^{\prime}(0)-C_{n-1,0}(0)+C_{n-1,0}^{\prime}(0) \\
=2 \cdot(-1)^{n-2}-c_{n-1,1,1}-0+(-1)^{n-1} .
\end{aligned}
$$

Thus, we have the recursive formula $c_{n, 1,1}=(-1)^{n}-c_{n-1,1,1}$. Starting from $C_{2,1}(q)=(q-1)^{3}$ and $c_{2,1,1}=3$, we obtain the closed form expression $c_{n, 1,1}=(-1)^{n}(n+1)$.

Concerning $C_{n, 2}(q)$, differentiate (3.8) with respect to $q$, obtaining

$$
C_{n, 2}^{\prime}(q)=C_{n-1,1}(q)+q \cdot C_{n-1,1}^{\prime}(q),
$$

whence

$$
c_{n, 2,1}=C_{n, 2}^{\prime}(0)=C_{n-1,1}(0), \quad \text { and } \quad c_{n, 2,1}=c_{n-1,1,0}=(-1)^{n-2} .
$$

Concerning $C_{n, 12}(q)$, differentiate (3.9) with respect to $q$, obtaining

$$
C_{n, 12}^{\prime}(q)=C_{n-1,1}(q)+(q-1) \cdot C_{n-1,1}^{\prime}(q)+C_{n-1,0}^{\prime}(q),
$$

whence

$$
\begin{aligned}
c_{n, 12,1}=C_{n, 12}^{\prime}(0) & =C_{n-1,1}(0)-C_{n-1,1}^{\prime}(0)+C_{n-1,0}^{\prime}(0) \\
& =c_{n-1,1,0}-c_{n-1,1,1}+c_{n-1,0,1}=(-1)^{n-2}-(-1)^{n-1} n+(-1)^{n-1}=(-1)^{n} n .
\end{aligned}
$$

At last, concerning $C_{n, 21}(q)$, we have from (3.10)

$$
\begin{aligned}
C_{n, 21}^{\prime}(q)=-C_{n-1,2}(q)-(q-1) & \cdot C_{n-1,2}^{\prime}(q) \\
& +C_{n-1,12}(q)+q \cdot C_{n-1,12}^{\prime}(q) \\
& -2(q-1) \cdot C_{n-1,21}(q)-(q-1)^{2} \cdot C_{n-1,21}^{\prime}(q),
\end{aligned}
$$

so, since $C_{n-1,2}(0)=0$ and $C_{n-1,21}(0)=0$,

$$
\begin{aligned}
c_{n, 21,1} & =C_{n, 21}^{\prime}(0)=-(-1) \cdot C_{n-1,2}^{\prime}(0)+C_{n-1,12}(0)-(-1)^{2} \cdot C_{n, 21}^{\prime}(0) \\
& =c_{n-1,2,1}+c_{n-1,12,0}-c_{n-1,21,1}=(-1)^{n-3}+(-1)^{n-2}-c_{n-1,21,1} \\
& =-c_{n-1,21,1} .
\end{aligned}
$$

Starting from $C_{2,21}(q)=q$ and $c_{2,21,1}=1$, we deduce $c_{n, 21,1}=(-1)^{n}$.

Theorem 3.7. The following identities are satisfied by the polynomials $C_{n,-}(q)$.

$$
\begin{gathered}
C_{n, 0}(q)=q^{2 n} C_{n, 0}\left(q^{-1}\right), \quad C_{n, 1}(q)=-q^{2 n-1} C_{n, 1}\left(q^{-1}\right), \quad C_{n, 2}(q)=-q^{2 n-1} C_{n, 2}\left(q^{-1}\right), \\
C_{n, 12}(q)=q^{2 n-2} C_{n, 12}\left(q^{-1}\right), \quad C_{n, 21}(q)=q^{2 n-2} C_{n, 21}\left(q^{-1}\right) .
\end{gathered}
$$

In terms of the coefficients of the various polynomials, we have

$$
\begin{gathered}
c_{n, 0, i}=c_{n, 0,2 n-i}, \quad c_{n, 1, i}=-c_{n, 1,2 n-1-i}, \quad c_{n, 2, i}=-c_{n, 2,2 n-1-i}, \\
c_{n, 12, i}=c_{n, 12,2 n-2-i}, \quad c_{n, 21, i}=c_{n, 21,2 n-2-i} .
\end{gathered}
$$


These identities reflect certain palindromic properties of the polynomials and permit us to compute their degrees in corollary 3.8. We will say a polynomial $p(x)=a_{0}+a_{1} x+\cdots+a_{n} x^{n}$ of degree $n$ is palindromic if

$$
p(x)=x^{n} p\left(x^{-1}\right)=x^{n}\left(a_{0}+a_{1} x^{-1}+\cdots+a_{n} x^{-n}\right)=a_{n}+a_{n-1} x+\cdots+a_{0} x^{n} .
$$

We say a polynomial of degree $n$ is skew-palindromic if $p(x)=-x^{n} p\left(x^{-1}\right)$. Obviously, for a palindromic or a skew-palindromic polynomial the leading and trailing coefficients are both non-vanishing.

Proof. These are all proved by induction, using the recursive formulas from theorem 3.2 . For the first identity,

$$
\begin{aligned}
C_{n, 0}(q) & =q^{2} \cdot C_{n-1,21}(q)-q(q-1) \cdot C_{n-1,1}(q) \\
& =q^{2} \cdot q^{2 n-4} C_{n-1,21}\left(q^{-1}\right)-q(q-1) \cdot\left(-q^{2 n-3} C_{n-1,1}\left(q^{-1}\right)\right) \\
& =q^{2 n} \cdot\left(q^{-2} C_{n-1,21}\left(q^{-1}\right)\right)-q^{2 n} \cdot\left(q^{-1}\left(q^{-1}-1\right) \cdot C_{n-1,1}\left(q^{-1}\right)\right)=q^{2 n} \cdot C_{n, 0}\left(q^{-1}\right) .
\end{aligned}
$$

For the second identity,

$$
\begin{aligned}
C_{n, 1}(q) & =-(q-1)^{2} \cdot C_{n-1,1}(q)-(q-1) \cdot C_{n-1,0}(q) \\
& =-(q-1)^{2} \cdot(-1) \cdot q^{2 n-3} C_{n-1,1}\left(q^{-1}\right)-(q-1) \cdot q^{2 n-2} C_{n-1,0}\left(q^{-1}\right) \\
& =q^{2 n-1} \cdot\left(\left(1-q^{-1}\right)^{2} \cdot C_{n-1,1}\left(q^{-1}\right)+\left(q^{-1}-1\right) \cdot C_{n-1,0}\left(q^{-1}\right)\right) \\
& =-q^{2 n-1} \cdot C_{n, 1}\left(q^{-1}\right) .
\end{aligned}
$$

For the third identity,

$$
\begin{aligned}
C_{n, 2}(q)=q \cdot C_{n-1,1}(q)=q \cdot(-1) \cdot q^{2 n-3} & \cdot C_{n-1,1}\left(q^{-1}\right) \\
& =-q^{2 n-1} \cdot\left(q^{-1} \cdot C_{n-1,1}\left(q^{-1}\right)=-q^{2 n-1} \cdot C_{n, 2}\left(q^{-1}\right) .\right.
\end{aligned}
$$

For the fourth identity,

$$
\begin{aligned}
C_{n, 12}(q) & =(q-1) \cdot C_{n-1,1}(q)+C_{n-1,0}(q) \\
& =(q-1) \cdot(-1) \cdot q^{2 n-3} \cdot C_{n-1,1}\left(q^{-1}\right)+q^{2 n-2} \cdot C_{n-1,0}\left(q^{-1}\right) \\
& \left.=q^{2 n-2} \cdot\left(q^{-1}-1\right) \cdot C_{n-1,1}\left(q^{-1}\right)+C_{n-1,0}\left(q^{-1}\right)\right) \\
& =q^{2 n-2} \cdot C_{n, 12}\left(q^{-1}\right) .
\end{aligned}
$$

Finally, for the fifth identity,

$$
\begin{aligned}
C_{n, 21}(q) & =-(q-1) \cdot C_{n-1,2}(q)+q \cdot C_{n-1,12}(q)-(q-1)^{2} \cdot C_{n-1,21}(q) \\
& =-(q-1) \cdot(-1) \cdot q^{2 n-3} \cdot C_{n-1,2}\left(q^{-1}\right)+q \cdot q^{2 n-4} \cdot C_{n-1,12}\left(q^{-1}\right) \\
& \quad-(q-1)^{2} \cdot q^{2 n-4} \cdot C_{n-1,21}\left(q^{-1}\right) \\
= & \left.q^{2 n-2} \cdot\left(q^{-1}-1\right)^{2} \cdot C_{n-1,2}\left(q^{-1}\right)+q^{-1} \cdot C_{n-1,12}\left(q^{-1}\right)-\left(q^{-1}-1\right)^{2} \cdot C_{n-1,21}\left(q^{-1}\right)\right) \\
= & q^{2 n-2} \cdot C_{n, 21}\left(q^{-1}\right) .
\end{aligned}
$$

Now we state the implications for the polyomials $C_{n, *}(q)$. 
Corollary 3.8. The degrees of the polynomials $C_{n, *}(q)$ are as follows.

$$
\begin{gathered}
\operatorname{deg}\left(C_{n, 0}\right)=2 n-1, \quad \operatorname{deg}\left(C_{n, 1}\right)=2 n-1, \quad \operatorname{deg}\left(C_{n, 2}\right)=2 n-2, \\
\operatorname{deg}\left(C_{n, 12}\right)=2 n-2, \quad \text { and } \quad \operatorname{deg}\left(C_{n, 21}\right)=2 n-3 .
\end{gathered}
$$

Proof. Consider first $C_{n, 0}(q)$. We know from proposition 3.5 that $C_{n, 0}(0)=0$, so $q$ is a factor. That is, $P_{n, 0}(q)=q^{-1} C_{n, 0}(q)$ is also a polynomial, and its trailing coefficient is $c_{n, 0,1} \neq 0$ by proposition 3.6. We observe

$$
P_{n, 0}(q)=q^{-1} C_{n, 0}(q)=q^{-1} \cdot q^{2 n} C_{n, 0}\left(q^{-1}\right)=q^{2 n-2} \cdot q C_{n, 0}\left(q^{-1}\right)=q^{2 n-2} \cdot P_{n, 0}\left(q^{-1}\right) .
$$

Thus, $C_{n, 0}(q)$ is $q$ times a palindromic polynomial of degree $2 n-2$, which means $C_{n, 0}(q)$ has degree $2 n-1$. Similarly, we conclude that $C_{n, 2}(q)$ and $C_{n, 21}(q)$ are, respectively, $q$ times a skew-palindromic polynomial of degree $2 n-3$ and $q$ times a palindromic polynomial of degree $2 n-4$. Thus $C_{n, 2}(q)$ has degree $2 n-2$ and $C_{n, 21}(q)$ has degree $2 n-3$. Since $C_{n, 1}(0) \neq 0$ and $C_{n, 12}(0) \neq 0$, the identities stated in theorem yield that $C_{n, 1}(q)$ is a skew-palindromic polynomial of degree $2 n-1$ and that $C_{n, 12}(q)$ is a palindromic polynomial of degree $2 n-2$.

Accordingly, set

$$
\begin{aligned}
& C_{n, 0}(q)=\sum_{i=1}^{2 n-1} c_{n, 0, i} q^{i}, \quad C_{n, 1}(q)=\sum_{i=0}^{2 n-1} c_{n, 1, i} q^{i}, \quad C_{n, 2}(q)=\sum_{i=1}^{2 n-2} c_{n, 2, i} q^{i}, \\
& C_{n, 12}(q)=\sum_{i=0}^{2 n-2} c_{n, 12, i} q^{i}, \quad \text { and } \quad C_{n, 21}(q)=\sum_{i=1}^{2 n-3} c_{n, 21, i} q^{i}
\end{aligned}
$$

\section{Obtaining the Polynomial Invariants}

Following the construction given in [6, p.288] we work over the function field $K=\mathbf{C}(q, z)$ and follow their recipes to obtain expressions for the two-variable HOMFLY-PT polynomials, the one-variable Jones polynomials $V_{W(3, n)}(t)$, and the Alexander polynomials $\Delta_{W(3, n)}(t)$. The expressions are subsequently refined to incorporate information obtained in section 3 . From this point we evaluate the span of the Jones polynomial $V_{W(3, n)}(t)$ in proposition 4.5, a result already known to Kauffman [7, Theorem 2.10], where we demonstrate how to use equations (3.27) and (3.30).

Let $H_{N+1}$ be the Hecke algebra over $K$ corresponding to $q$ with $N$ generators as in definition 3.1. The starting point is the following theorem.

Theorem 4.1. For $N \geq 1$ there is a family of trace functions $\operatorname{Tr}: H_{N+1} \rightarrow K$ compatible with the inclusions $H_{N} \rightarrow H_{N+1}$ satisfying

1. $\operatorname{Tr}(1)=1$,

2. $\operatorname{Tr}$ is $K$-linear and $\operatorname{Tr}(a b)=\operatorname{Tr}(b a)$,

3. If $a, b \in H_{N}$, then $\operatorname{Tr}\left(a T_{N} b\right)=z \operatorname{Tr}(a b)$. 
Property 3 enables the calculation of $\mathrm{Tr}$ on basis elements of $H_{N+1}$ through use of the defining relations and induction. For $\mathrm{H}_{3}$, note that

$\operatorname{Tr}\left(T_{1}\right)=\operatorname{Tr}\left(T_{2}\right)=z, \quad \operatorname{Tr}\left(T_{1} T_{2}\right)=\operatorname{Tr}\left(T_{2} T_{1}\right)=z^{2}, \quad \operatorname{Tr}\left(T_{1} T_{2} T_{1}\right)=z \operatorname{Tr}\left(T_{1}^{2}\right)=z((q-1) z+q)$,

and we put $w=1-q+z$. The next step toward the polynomial invariants of the knot that is the closure of the braid $\alpha \in B_{N+1}$ is given by the formula

$$
V_{\alpha}(q, z)=\left(\frac{1}{z}\right)^{(N+e(\alpha)) / 2} \cdot\left(\frac{q}{w}\right)^{(N-e(\alpha)) / 2} \cdot \operatorname{Tr}(\rho(\alpha)),
$$

where $e(\alpha)$ is the exponent sum of the word $\alpha$. The expression defines an element in the quadratic extension $K(\sqrt{q / z w})$. For the weaving knot $W(3, n)$, viewed as the closure of $\left(\sigma_{1} \sigma_{2}^{-1}\right)^{n}$, we have the exponent sum $e=0$, and $N=2$, and

$\rho\left(\left(\sigma_{1} \sigma_{2}^{-1}\right)^{n}\right)=\left(T_{1} T_{2}^{-1}\right)^{n}=q^{-n}\left(C_{n, 0}(q)+C_{n, 1}(q) \cdot T_{1}+C_{n, 2}(q) \cdot T_{2}+C_{n, 12}(q) \cdot T_{1} T_{2}+C_{n, 21}(q) \cdot T_{2} T_{1}\right)$,

thanks to proposition 3.4, which says the expression for $\left(T_{1} T_{2}^{-1}\right)^{n}$ requires only the use of the basis elements $1, T_{1}, T_{2}, T_{1} T_{2}$ and $T_{2} T_{1}$. Then we have

$$
\begin{aligned}
& V_{\left(\sigma_{1} \sigma_{2}^{-1}\right)^{n}}(q, z) \\
= & \left(\frac{1}{z}\right) \cdot\left(\frac{q}{w}\right) \cdot q^{-n} \operatorname{Tr}\left(C_{n, 0}(q)+C_{n, 1}(q) \cdot T_{1}+C_{n, 2}(q) \cdot T_{2}+C_{n, 12}(q) \cdot T_{1} T_{2}+C_{n, 21}(q) \cdot T_{2} T_{1}\right) \\
& =\left(\frac{q}{z w}\right) \cdot q^{-n} \cdot\left(C_{n, 0}(q)+C_{n, 1}(q) \cdot z+C_{n, 2}(q) \cdot z+C_{n, 12}(q) \cdot z^{2}+C_{n, 21}(q) \cdot z^{2}\right), \quad(4.1)
\end{aligned}
$$

using the facts that $\operatorname{Tr} T_{1}=\operatorname{Tr} T_{2}=z$ and $\operatorname{Tr} T_{1} T_{2}=\operatorname{Tr} T_{2} T_{1}=z^{2}$. This expression is the starting point for our manipulations.

Following [6], we point out that the universal skein invariant $P_{W(3, n)}(\ell, m)$, an element of the Laurent polynomial ring $\mathbf{Z}\left[\ell, \ell^{-1}, m, m^{-1}\right]$, is obtained by rewriting $V_{\left(\sigma_{1} \sigma_{2}^{-1}\right)^{n}}(q, z)$ in terms of $\ell=i(z / w)^{1 / 2}$ and $m=i\left(q^{-1 / 2}-q\right)$, a task easily managed in a computer algebra system by simplifying $V_{\left(\sigma_{1} \sigma_{2}^{-1}\right)^{n}}(q, z)$ with respect to side relations. Starting from $P_{W(3, n)}(\ell, m)$, the Jones polynomial $V_{W(3, n)}(t)$ is obtained by setting $\ell=i t$ and $m=i\left(t^{1 / 2}-t^{-1 / 2}\right)$, the Alexander polynomial $\Delta_{W(3, n)}(t)$ is obtained by setting $\ell=i$ and $m=i\left(t^{1 / 2}-t^{-1 / 2}\right)$, and the HOMFLY-PT polynomial is obtained by setting $\ell=i a$ and $m=i z$. We have no specific use for the HOMFLY-PT polynomial in this paper, so we content ourselves with a few values in section 9 .

To obtain the Alexander polynomial from $V_{\left(\sigma_{1} \sigma_{2}^{-1}\right)^{n}}(q, z)$, it is useful to first rewrite

$$
V_{\left(\sigma_{1} \sigma_{2}^{-1}\right)^{n}}(q, z)=q^{-n+1} \cdot\left(C_{n, 0}(q) \cdot(z w)^{-1}+\left(C_{n, 1}(q)+C_{n, 2}(q)\right) \cdot w^{-1}+\left(C_{n, 12}(q)+C_{n, 21}(q)\right) \cdot z w^{-1}\right) .
$$

First set $q=t$ and make the substitutions

$$
z=\frac{\ell^{2}(t-1)}{1+\ell^{2}}, \quad w=1-q+z=1-t+z=\frac{-1(t-1)}{1+\ell^{2}}
$$

to obtain an expression

$$
\begin{aligned}
t^{-n+1} \cdot\left(C_{n, 0}(t) \cdot\left(\frac{\left(1+\ell^{2}\right)}{\ell^{2}}\right)^{2} \cdot \frac{1}{(t-1) t}+\left(C_{n, 1}(t)+C_{n, 2}(t)\right) \cdot \frac{\left.(-1) \dot{(1}+\ell^{2}\right)}{t-1}\right. \\
\left.+\left(C_{n, 12}(t)+C_{n, 21}(t)\right) \cdot(-1) \cdot \ell^{2}\right)
\end{aligned}
$$


Now make the substitution $\ell=i$ and we arrive at

$$
\Delta_{W(3, n)}(t)=t^{-n+1}\left(C_{n, 12}(t)+C_{n, 21}(t)\right) .
$$

Evidently a lot of information from the braid representation of $W(3, n)$ has been lost. To see what remains, corollary 3.8 says that the degree of $C_{n, 12}(t)$ is $2 n-2$ and the degree of $C_{n, 21}(t)$ is $2 n-3$. It follows that the degree of $\Delta_{W(3, n)}(t)$ is $(2 n-2)-n+1=n-1$. Moreover, the lowest order non-vanishing coefficients are $c_{n, 12,0}=(-1)^{n}$ and $c_{n, 21,1}=(-1)^{n}$. By theorem 3.7 we also have $c_{n, 12,2 n-2}=c_{n, 12,0}=(-1)^{n}$ and $c_{n, 21,2 n-3}=c_{n, 21,1}=(-1)^{n}$. Thus,

$$
\Delta_{W(3, n)}(t)=a_{0}+\sum_{s>0} a_{s}\left(t^{s}+t^{-s}\right)=(-1)^{n} \cdot t^{-n+1}+\cdots+(-1)^{n} \cdot t^{n-1} .
$$

Theorem 4.2. The Seifert genus of $W(3, n)$ is $n-1$, and the complement of $W(3, n)$ is fibered over $S^{1}$.

Proof. We know the signature of $W(3, n)$ is zero, by Proposition 2.4, so we apply [13, Theorem 1.3] relating the coefficients of the Alexander polynomial and the signature of $W(3, n)$ to the ranks of the Heegard-Floer homology groups of $S^{3}$ associated to $W(3, n)$. The result is

$$
\widehat{H F K}_{s}\left(S^{3}, W(3, n), s\right)=\left\{\begin{array}{l}
\mathbf{Z}^{\left|a_{s}\right|}, \quad 0 \leq s \leq n-1, \\
0, \quad \text { else. }
\end{array}\right.
$$

By [14, Theorem 1.2], Seifert genus of $W(3, n)$ is $n-1$. Since we have explicitly

$$
\widehat{H F K}_{s}\left(S^{3}, W(3, n), n-1\right) \cong \mathbf{Z}
$$

[10, Theorem 2.5] says that the complement of $W(3, n)$ is fibered over $S^{1}$.

Turning to the Jones polynomial, we follow a similar scheme, but the details are necessarily more complicated. To start, the substitutions

$$
q=t, \quad z=\frac{t^{2}}{1+t}, \quad w=\frac{1}{1+t}
$$

in (4.1) lead to the one-variable Jones polynomial

$$
\begin{gathered}
V_{W(3, n)}(t)=\frac{t(1+t)^{2}}{t^{2}} \cdot t^{-n} \cdot\left(C_{n, 0}(t)+\left(C_{n, 1}(t)+C_{n, 2}(t)\right) \cdot \frac{t^{2}}{1+t}+\left(C_{n, 12}(t)+C_{n, 21}(t)\right) \cdot \frac{t^{4}}{(1+t)^{2}}\right) \\
=t^{-n-1} \cdot\left((1+t)^{2} \cdot C_{n, 0}(t)+(1+t) \cdot\left(C_{n, 1}(t)+C_{n, 2}(t)\right) \cdot t^{2}+\left(C_{n, 12}(t)+C_{n, 21}(t)\right) \cdot t^{4}\right) .
\end{gathered}
$$

Example 4.3. For $W(3,1)$, which is the unknot, we have

$$
\begin{aligned}
V_{W(3,1)}(t) & =t^{-2} \cdot\left((1+t)^{2} \cdot C_{1,0}(t)+(1+t) \cdot\left(C_{1,1}(t)+C_{1,2}(t)\right) \cdot t^{2}+\left(C_{1,12}(t)+C_{1,21}(t)\right) \cdot t^{4}\right) \\
& =t^{-2} \cdot\left((1+t)^{2} \cdot 0+(1+t) \cdot(-(t-1)+0) \cdot t^{2}+(1+0) \cdot t^{4}\right) \\
& =t^{-2} \cdot\left(\left(1-t^{2}\right) t^{2}+t^{4}\right)=1 .
\end{aligned}
$$


Example 4.4. For $W(3,2)$, which is the figure- 8 knot, we have

$$
\begin{aligned}
V_{W(3,2)}(t) & =t^{-3} \cdot\left((1+t)^{2} \cdot C_{2,0}(t)+(1+t) \cdot\left(C_{2,1}(t)+C_{2,2}(t)\right) \cdot t^{2}+\left(C_{2,12}(t)+C_{2,21}(t)\right) \cdot t^{4}\right) \\
& =t^{-3} \cdot\left((1+t)^{2} \cdot t(t-1)^{2}+(1+t) \cdot\left((t-1)^{3}-t(t-1)\right) \cdot t^{2}+\left(-(t-1)^{2}+t\right) \cdot t^{4}\right) \\
& =t^{-3} \cdot\left(t^{5}-t^{4}+t^{3}-t^{2}+t\right)=t^{2}-t+1-t^{-1}+t^{-2}
\end{aligned}
$$

Now we take a closer look at the formal expression

$$
\begin{aligned}
& V_{W(3, n)}(t)= \\
& \quad=t^{-n-1} \cdot\left((1+t)^{2} \cdot C_{n, 0}(t)+(1+t) \cdot\left(C_{n, 1}(t)+C_{n, 2}(t)\right) \cdot t^{2}+\left(C_{n, 12}(t)+C_{n, 21}(t)\right) \cdot t^{4}\right)
\end{aligned}
$$

for the Jones polynomial of the weaving knot $W(3, n)$.

Incorporating the formal expansions given in equations (3.32), we have

$$
\begin{gathered}
V_{W(3, n)}(t)=t^{-n-1} \cdot\left((1+t)^{2} \cdot C_{n, 0}(t)+\left(t^{2}+t^{3}\right) \cdot\left(C_{n, 1}(t)+C_{n, 2}(t)\right)+t^{4} \cdot\left(C_{n, 12}(t)+C_{n, 21}(t)\right)\right) \\
=t^{-n-1} \cdot\left((1+t)^{2} \cdot\left(\sum_{i=1}^{2 n-1} c_{n, 0, i} t^{i}\right)\right. \\
\quad+\left(t^{2}+t^{3}\right) \cdot\left(\sum_{i=0}^{2 n-1} c_{n, 1, i} t^{i}+\sum_{i=1}^{2 n-2} c_{n, 2, i} t^{i}\right) \\
\left.\quad+t^{4} \cdot\left(\sum_{i=0}^{2 n-2} c_{n, 12, i} t^{i}+\sum_{i=1}^{2 n-3} c_{n, 21, i} t^{i}\right)\right) \\
=t^{-n-1} \cdot P(t)=t^{-n-1} \cdot\left(p_{0}+p_{1} t+p_{2} t^{2}+p_{3} t^{3}+p_{4} t^{4}+\cdots\right)
\end{gathered}
$$

The first piece of information about the Jones polynomial $V_{W(3, n)}(t)$ we obtain by using results about the $C_{n,-}$ is the following fact, due to Kauffmann [7, Theorem 2.10].

Proposition 4.5. The span of the Jones polynomial $V_{W(3, n)}(t)$ is $2 n$, and the trailing and leading coefficients are $(-1)^{n}$.

Proof. We observe that $p_{0}=P(0)=1 \cdot c_{n, 0,0}=0$ by proposition 3.5 , so the lowest non-zero term in $V_{W(3, n)}(t)$ is $t^{-n-1} \cdot p_{1} t$. Clearly, $p_{1}=c_{n, 0,1}=(-1)^{n}$ by proposition 3.6.

One identifies the top degree term in the polynomial factor of (4.4) as the term of degree $2 n+2$ with coefficient

$$
c_{n, 1,2 n-1}+c_{n, 12,2 n-2}=-c_{n, 1,0}+c_{n, 12,0}=-(-1)^{n-1}+(-1)^{n-1}=0,
$$

where we use the palindromic equations (3.30) and the table of trailing coefficients (3.27) to do the computation.

Turning to the term of degree $2 n+1$, we find the coefficient is

$$
\begin{aligned}
c_{n, 0,2 n-1} & +c_{n, 1,2 n-1}+c_{n, 1,2 n-2}+c_{n, 2,2 n-2}+c_{n, 12,2 n-3}+c_{n, 21,2 n-3} \\
& =c_{n, 0,1}-c_{n, 1,0}-c_{n, 1,1}-c_{n, 2,1}+c_{n, 12,1}+c_{n, 21,1} \\
& =(-1)^{n}-(-1)^{n-1}-(-1)^{n}(n+1)-(-1)^{n}+(-1)^{n} n+(-1)^{n} \\
& =(-1)^{n}
\end{aligned}
$$

using first (3.30) and then the tables (3.27) and (3.28) to complete the evaluation. 
Thus, we may write the Jones polynomial in the form

$$
V_{W(3, n)}(t)=(-1)^{n} t^{-n}+v_{-n+1} t^{-n+1}+v_{-n+2} t^{-n+2}+\cdots+v_{n-2} t^{n-2}+v_{n-1} t^{n-1}+(-1)^{n} t^{n},
$$

where it is known that $v_{-n+i}=v_{n-i}$, since $W(p, q)$ for $p$ odd is amphicheiral. The twist number of $W(3, n)$ is $\left|v_{-n+1}\right|+\left|v_{n-1}\right|$ according to [5]. We will now recompute the twist number for $n \geq 3$ from the information we have gathered about the coefficients of the Jones polynomial. First, observe that

$$
v_{-n+1} t^{-n+1}=t^{-n-1} \cdot p_{2} t^{2}
$$

and $p_{2} t^{2}$ is computed from

$$
(1+2 t) \cdot\left(c_{n, 0,1} t+c_{n, 0,2} t^{2}\right)+t^{2} \cdot c_{n, 1,0}=c_{n, 0,1} t+\left(c_{n, 0,2}+2 c_{n, 0,1}+c_{n, 1,0}\right) \cdot t^{2},
$$

and no other terms from the expansion (4.4), because $c_{n, 2,0}=0$ by proposition 3.5. We have

$$
p_{2}=c_{n, 0,2}+2 c_{n, 0,1}+c_{n, 1,0}=c_{n, 0,2}+2 \cdot(-1)^{n}+(-1)^{n-1}
$$

by propositions 3.5 and 3.6 .

$$
=c_{n, 0,2}+(-1)^{n} .
$$

We identify $c_{n, 0,2}$ by reducing the recursive description (3.6) mod $q^{3}$, obtaining

$$
\begin{aligned}
c_{n, 0,0}+c_{n, 0,1} q+c_{n, 0,2} q^{2} & \equiv q^{2} \cdot\left(c_{n-1,21,0}\right)+\left(-q^{2}+q\right) \cdot\left(c_{n-1,1,0}+c_{n-1,1,1} q\right) \\
& \equiv c_{n-1,1,0} q+\left(c_{n-1,21,0}+c_{n-1,1,1}-c_{n-1,1,0}\right) \cdot q^{2} \bmod q^{3}
\end{aligned}
$$

After extracting the coefficient of $q^{2}$,

$$
\begin{aligned}
c_{n, 0,2} & =c_{n-1,21,0}+c_{n-1,1,1}-c_{n-1,1,0} \\
& =0+(-1)^{n-1}((n-1)+1)-(-1)^{n-2}=(-1)^{n-1}(n+1), \quad \text { for } n \geq 3,
\end{aligned}
$$

by propositions 3.5 and 3.6. Since we have used the formula for $c_{n-1,1,1}$ in (3.28), we must assume $n-1 \geq 2$. Referring to (4.6),

$$
v_{-n+1}=p_{2}=c_{n, 0,2}+(-1)^{n}=(-1)^{n-1} n+(-1)^{n-1}+(-1)^{n}=(-1)^{n-1} n .
$$

Thus, we have reproved the following formula given in theorem 5.1 of [5].

Proposition 4.6. For $n \geq 3$, the twist number of $W(3, n)$ is $\left|v_{-n+1}\right|+\left|v_{n-1}\right|=n+n=2 n$.

\section{Higher Twist Numbers and Volume}

In [5], Dasbach and Lin define higher twist numbers of a knot in terms of the Jones polynomial, with the idea that these invariants also correlate with the hyperbolic volume of the knot complement. If

$$
V_{K}(t)=\lambda_{-m} t^{-m}+\lambda_{-m+1} t^{-m+1}+\cdots+\lambda_{n-1} t^{n-1}+\lambda_{n} t^{n},
$$


then the $j$ th twist number of $K$ is $T_{j}(K)=\left|\lambda_{-m+j}\right|+\left|\lambda_{n-j}\right|$. Note that twist numbers $T_{j}(K)$ are only defined for $j$ within the span of the Jones polynomial. In the case of weaving knots $W(3, n)$, the relevant twist numbers are defined for $1 \leq j \leq n-1$. In proposition 4.6 we have recomputed the first twist number of $W(3, n)$ using our results from section 3 . In theorems 5.1 and 5.2 we extend the technique to compute the second and third twist numbers.

In the appendix to [5] one finds a scatter plot generated from a table of alternating knots of 14 crossings by plotting along a horizontal axis the higher twist numbers of the knots and along the vertical axis the volume of the complement. The authors also construct similar plots starting from a table of non-alternating knots of 14 crossings. In both cases, there appears to be some correlation between these combinatorial invariants and the geometric invariant. We are going to explore how well higher twist numbers and volume correlate as the number of crossings increases.

We supplement our rigorous calculations of $T_{2}(W(3, n))$ and $T_{3}(W(3, n))$ with some conjectural calculations in the following table of higher twist numbers. To obtain these results, we use Mathematica or Maple to extract the coefficients $\lambda_{-n+k}$ of $t^{-n+k}$ in $V_{W(3, n)}(t)$ for $k=4$, 5,6 , and 7 associated to weaving knots $W(3, n)$ starting near $n=2 k$. We conjecture that the $k$ th twist number $T_{k}(W(3, n))$ is a polynomial in $n$ of degree $k$. Taking iterated differences of the coefficient sequences, we find they are consistent with the conjecture as long as $n$ is sufficiently large. The following formulas for the twist numbers $T_{k}(W(3, n))=2\left|\lambda_{-n+k}\right|$ for $k=4,5,6$, and 7 were produced by fitting polynomials to sufficiently large selections of coefficients $\lambda_{-n+k}$ and comparing polynomial values with computed coefficients for different values of $n$. Figures 8 , 9, and 10 plot horizontally values of the twist numbers $T_{2}, T_{3}$, and

Table 1: Higher twist numbers for $W(3, n)$

\begin{tabular}{|c|l|}
\hline$k$ & $T_{k}(W(3, n))$ \\
\hline 2 & $-n+n^{2}$ \\
\hline 3 & $n(n-1)(n-2) / 3+2 n$ \\
\hline 4 & $-(9 / 2) n+(35 / 12) n^{2}-(1 / 2) n^{3}+(1 / 12) n^{4}$ \\
\hline 5 & $(42 / 5) n-(35 / 6) n^{2}+(19 / 12) n^{3}-(1 / 6) n^{4}+(1 / 60) n^{5}$ \\
\hline 6 & $-(52 / 3) n+(2237 / 180) n^{2}-(29 / 8) n^{3}+(41 / 72) n^{4}-(1 / 24) n^{5}+(1 / 360) n^{6}$ \\
\hline 7 & $\begin{array}{c}(254 / 7) n-(413 / 15) n^{2}+(1541 / 180) n^{3} \\
\end{array}$ \\
\hline
\end{tabular}

the conjectured $T_{4}$ and vertically values of the volume of the link complement. We used the program SnapPy [4] to compute estimates of the volume.

In addition, one can ask how efficient are the bounds given in (1.1) for the volume of weaving knots $W(p, q)$. For weaving knots $W(3, n)$ the bounds simplify to

$$
v_{\text {oct }} n\left(1-\frac{(2 \pi)^{2}}{n^{2}}\right)^{3 / 2} \leq \operatorname{vol}(W(3, n))<4 v_{\text {tet }} \cdot n .
$$

If we consider the volume relative to the crossing number $\operatorname{vol}(W(3, n)) / 2 n$, then we have the 
chain

$$
\frac{v_{\text {oct }}}{2}\left(1-\frac{(2 \pi)^{2}}{n^{2}}\right)^{3 / 2} \leq \frac{\operatorname{vol}(W(3, n))}{2 n}<2 v_{\text {tet }}
$$

For a fixed value of $n$ there is a gap between the upper and lower bounds. We can ask whether or not better bounds on the relative volume of weaving knots $W(3, n)$ can be teased out of the higher twist numbers of these knots. To obtain some information on the question we appeal to algorithms in the program SnapPy to generate estimates of the volume of these knots.

We perform the following manipulations on the formula for $T_{k}(W(3, n))$. First take the $k$ th root of the expression and then divide by the crossing number $2 n$ to obtain an expression whose limit as $n$ tends to infinity is finite. Then multiply by a normalization constant $C_{k}$ so that

$$
\lim _{n \rightarrow \infty} C_{k} \frac{\sqrt[k]{T_{k}(W(3, n))}}{2 n}=2 v_{\text {tet }}
$$

In figure 7 we show the upper bound from equation (5.1) as a horizontal line at the top of the plot and the lower bound as the lowest curve. Values vol $(W(3, n)) / 2 n$ according to SnapPy are plotted as points. We also plot $C_{k} \cdot \sqrt[k]{T_{k}(W(3, n))} / 2 n$ for $k=2,3$, and 4 . We see that all three of these curves provide better lower bounds on the relative volume than the lower bound given in (5.1). Indeed, for $n$ sufficiently large the lower bound from $T_{2}$ is better than the bound from $T_{3}$, which is, in turn, better than the bound from $T_{4}$.

Theorem 5.1. For $n \geq 5$, the second twist number of $W(3, n)$ is $n(n-1)$.

Proof. Comparing (4.4) with (4.5), and noting that $C_{n, 12}(t)$ and $C_{n, 21}(t)$ start in degrees 0 and 1 , respectively, we want to compute the term $p_{3} t^{3}$ from the truncated expansion

$$
\begin{aligned}
& p_{1} t+p_{2} t^{2}+p_{3} t^{3} \\
= & (1+t)^{2}\left(c_{n, 0,0}+c_{n, 0,1} t+c_{n, 0,2} t^{2}+c_{n, 0,3} t^{3}\right)+\left(t^{2}+t^{3}\right)\left(\left(c_{n, 1,0}+c_{n, 1,1} t\right)+\left(c_{n, 2,0}+c_{n, 2,1} t\right)\right) .
\end{aligned}
$$

Extracting the coefficient of $t^{3}$ and substituting from (3.27) and (3.28) as well as equation (4.7) yields

$$
\begin{gathered}
p_{3}=\left(c_{n, 0,3}+2 c_{n, 0,2}+c_{n, 0,1}\right)+\left(c_{n, 1,0}+c_{n, 1,1}\right)+\left(c_{n, 2,0}+c_{n, 2,1}\right) \\
=\left(c_{n, 0,3}+2(-1)^{n-1}(n+1)+(-1)^{n}\right)+\left((-1)^{n-1}+(-1)^{n}(n+1)\right)+\left(0+(-1)^{n}\right) \\
=c_{n, 0,3}+(-1)^{n-1} n .
\end{gathered}
$$

We reduce the recursive formula (3.6) modulo $t^{4}$ to compute $c_{n, 0,3}$.

$$
\begin{aligned}
C_{n, 0}(t)= & t^{2} \cdot C_{n-1,21}(t)-t(t-1) \cdot C_{n-1,1}(t) \\
\equiv & t^{2}\left(c_{n-1,21,0}+c_{n-1,21,1} t\right)-t^{2}\left(c_{n-1,1,0}+c_{n-1,1,1} t\right)+t\left(c_{n-1,1,0}+c_{n-1,1,1} t+c_{n-1,1,2} t^{2}\right) \\
& \quad \bmod t^{4}
\end{aligned}
$$

so after extracting the coefficient of $t^{3}$, we have

$$
\begin{aligned}
c_{n, 0,3}=c_{n-1,21,1}-c_{n-1,1,1} & +c_{n-1,1,2} \\
& =(-1)^{n-1}-(-1)^{n-1} n+c_{n-1,1,2}=(-1)^{n}(n-1)+c_{n-1,1,2} .
\end{aligned}
$$




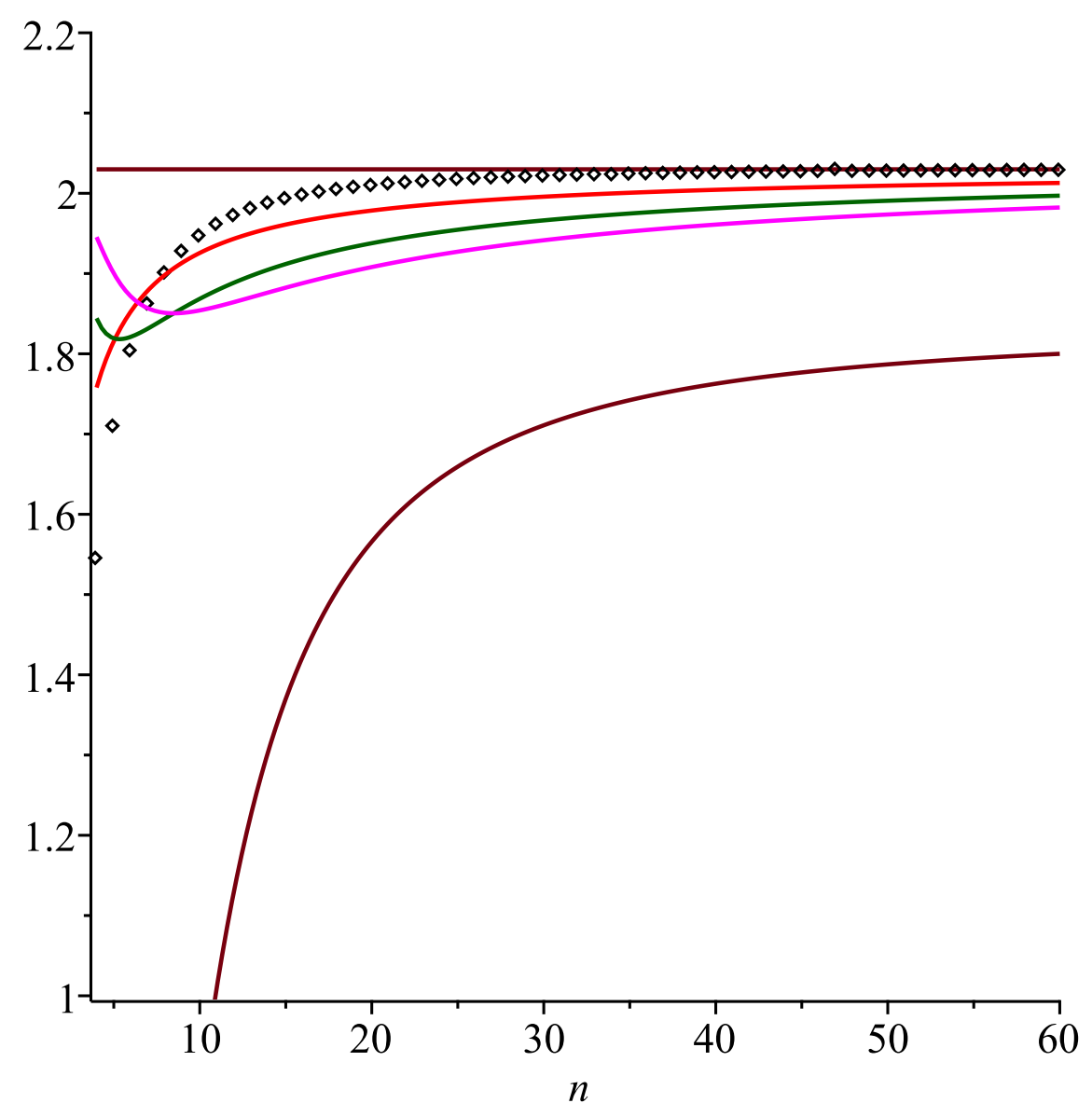

Figure 7: Comparing bounds

Thus, we need a formula for $c_{n-1,1,2}$. For this return to the recursive formula (3.7) and differentiate twice, obtaining

$C_{n, 1}^{(2)}(q)=-2 \cdot C_{n-1,1}(q)-4(q-1) \cdot C_{n-1,1}^{\prime}(q)-(q-1)^{2} \cdot C_{n-1,1}^{(2)}(q)-2 C_{n-1,0}^{\prime}(q)-(q-1) \cdot C_{n-1,0}^{(2)}(q)$.

Substituting $q=0$, we get

$$
\begin{aligned}
2 c_{n, 1,2} & =-2 c_{n-1,1,0}+4 c_{n-1,1,1}-2 c_{n-1,1,2}-2 c_{n-1,0,1}+2 c_{n-1,0,2}, \\
& =-2 \cdot(-1)^{n-2}+4 \cdot(-1)^{n-1} n-2 c_{n-1,1,2}-2(-1)^{n-1}+2(-1)^{n-2} n,
\end{aligned}
$$

applying propositions 3.5 and 3.6 and formula (4.7). Note that the use of (4.7) requires $n-1 \geq 3$, so we have to have $n \geq 4$. Rewriting this expression, we get

$$
c_{n, 1,2}+c_{n-1,1,2}=(-1)^{n-1} n, \quad \text { for } n \geq 4 .
$$


Now we create a closed form expression for $c_{n, 1,2}$ by forming a telescoping sum.

$$
\begin{aligned}
c_{n, 1,2}+c_{n-1,1,2} & =(-1)^{n-1} n \\
-c_{n-1,1,2}-c_{n-2,1,2} & =(-1)^{n-1}(n-1) \\
& \cdots \\
(-1)^{k} c_{n-k, 1,2}+(-1)^{k} c_{n-k-1,1,2} & =(-1)^{k}(-1)^{n-k-1}(n-k) \\
& \cdots \\
(-1)^{n-5} c_{5,1,2}+(-1)^{n-5} c_{4,1,2} & =(-1)^{n-1} 5 \\
(-1)^{n-4} c_{4,1,2}+(-1)^{n-4} c_{3,1,2} & =(-1)^{n-1} 4 .
\end{aligned}
$$

Adding these equations yields

$$
\begin{aligned}
c_{n, 1,2}+(-1)^{n-4} c_{3,1,2}= & (-1)^{n-1} \sum_{k=4}^{n} k \\
& =(-1)^{n-1}\left(\frac{n(n+1)}{2}-\frac{3(3+1)}{2}\right)=(-1)^{n-1}(n(n+1) / 2-6) .
\end{aligned}
$$

From section 9, $C_{3,1}(q)=1-4 q+7 q^{2}-7 q^{3}+4 q^{4}-q^{5}$, so $c_{3,1,2}=7$ and we obtain

$$
c_{n, 1,2}=(-1)^{n-1}\left(\frac{n(n+1)}{2}+1\right), \quad \text { for } n \geq 4 .
$$

Substituting the results of (5.3) and (5.5) into (5.2), we obtain

$$
\begin{aligned}
v_{-n+2}=p_{3} & =(-1)^{n-1} n+c_{n, 0,3} \\
& =(-1)^{n-1} n+\left((-1)^{n}(n-1)+c_{n-1,1,2}\right) \\
& =\left((-1)^{n-1} n+(-1)^{n}(n-1)\right)+(-1)^{n-2}(n-1) n / 2+(-1)^{n-2} \\
& =(-1)^{n}(n-1) n / 2 .
\end{aligned}
$$

for $n-1 \geq 4$, or $n \geq 5$. We conclude the second twist number for $W(3, n)$ is $\left|v_{-n+2}\right|+\left|v_{n-2}\right|=$ $n(n-1)$.

Theorem 5.2. For $n \geq 5$ the coefficient of $t^{-n+3}$ in the Jones polynomial $V_{W(3, n)}(t)$ is

$$
v_{-n+3}=(-1)^{n-1}(n(n-1)(n-2) / 6+n),
$$

so third twist number for $W(3, n)$ is $\left|v_{-n+3}\right|+\left|v_{n-3}\right|=n(n-1)(n-2) / 3+2 n$.

Proof. The essential point is to compute the coefficient $p_{4}$ in the expansion (4.5). Starting from the truncated polynomial expression

$$
\begin{aligned}
\left(1+2 t+t^{2}\right)\left(c_{n, 0,1} t+c_{n, 0,2} t^{2}+c_{n, 0,3} t^{3}+c_{n, 0,4} t^{4}\right) & \\
+\left(t^{2}+t^{3}\right)\left[\left(c_{n, 1,0}+c_{n, 1,1} t+c_{n, 1,2} t^{2}\right)+\left(c_{n, 2,0}+c_{n, 2,1} t+\right.\right. & \left.\left.c_{n, 2,2} t^{2}\right)\right] \\
& +t^{4}\left(c_{n, 12,0}+c_{n, 21,0}\right)
\end{aligned}
$$


we extract the coefficient of $t^{4}$, obtaining

$$
\begin{aligned}
p_{4} & =\left(c_{n, 0,4}+2 c_{n, 0,3}+c_{n, 0,2}\right)+\left[\left(c_{n, 1,1}+c_{n, 1,2}\right)+\left(c_{n, 2,1}+c_{n, 2,2}\right)\right]+\left(c_{n, 12,0}+c_{n, 21,0}\right) \\
& =\left(c_{n, 0,4}+2 c_{n, 0,3}+c_{n, 0,2}\right)+\left[\left((-1)^{n}(n+1)+c_{n, 1,2}\right)+\left((-1)^{n}+c_{n, 2,2}\right)\right]+(-1)^{n-1}
\end{aligned}
$$

by (3.27) and (3.28),

$$
\left.=\left(c_{n, 0,4}+2 c_{n, 0,3}+(-1)^{n-1}(n+1)\right)+\left[\left((-1)^{n}(n+1)+c_{n, 1,2}\right)+c_{n, 2,2}\right)\right]
$$

evaluating $c_{n, 0,2}=(-1)^{n-1}(n+1)$ for $n \geq 3$ by (4.7),

$$
\begin{aligned}
& =c_{n, 0,4}+2 c_{n, 0,3}+c_{n, 1,2}+c_{n, 2,2} \\
& =c_{n, 0,4}+2\left((-1)^{n}(n-1)+c_{n-1,1,2}\right)+c_{n, 1,2}+c_{n-1,1,1}
\end{aligned}
$$

substituting for $c_{n, 0,3}$ from (5.3) and using (3.8) which implies $c_{n, 2,2}=c_{n-1,1,1}$,

$$
=c_{n, 0,4}+2(-1)^{n}(n-1)+c_{n-1,1,2}+(-1)^{n-1} n+(-1)^{n-1} n
$$

since $c_{n, 1,2}+c_{n-1,1,2}=(-1)^{n-1} n$ by (5.4) and $c_{n-1,1,1}=(-1)^{n-1} n$ by (3.28),

$$
=c_{n, 0,4}+c_{n-1,1,2}+2(-1)^{n-1}
$$

Compute $c_{n, 0,4}$ from the recursion formula (3.6) reduced modulo $q^{5}$, which yields

$$
\begin{aligned}
& c_{n, 0,0}+c_{n, 0,1} q+c_{n, 0,2} q^{2}+c_{n, 0,3} q^{3}+c_{n, 0,4} q^{4} \\
&=q^{2}\left(c_{n-1,21,0}+c_{n-1,21,1} q+c_{n-1,21,2} q^{2}\right)-q^{2}\left(c_{n-1,1,0}+c_{n-1,1,1} q+c_{n-1,1,2} q^{2}\right) \\
&+q\left(c_{n-1,1,0}+c_{n-1,1,1} q+c_{n-1,1,2} q^{2}+c_{n-1,1,3} q^{3}\right)
\end{aligned}
$$

Extracting the coefficients of $q^{4}$ gives

$$
c_{n, 0,4}=c_{n-1,21,2}-c_{n-1,1,2}+c_{n-1,1,3}
$$

so we have

$$
\begin{aligned}
p_{4} & =c_{n, 0,4}+c_{n-1,1,2}+2(-1)^{n-1}=\left(c_{n-1,21,2}-c_{n-1,1,2}+c_{n-1,1,3}\right)+c_{n-1,1,2}+2(-1)^{n-1} \\
& =c_{n-1,21,2}+c_{n-1,1,3}+2(-1)^{n-1}
\end{aligned}
$$

We now deal with $c_{n-1,21,2}$ by reducing the recurrence relation (3.10) $\bmod q^{3}$. We get

$$
\begin{aligned}
c_{n, 21,0}+c_{n, 21,1} q+c_{n, 21,2} q^{2} & \\
\equiv(-q+1)\left(c_{n-1,2,0}+c_{n-1,2,1} q+c_{n-1,2,2} q^{2}\right)+q\left(c_{n-1,12,0}+c_{n-1,12,1} q\right) & \\
-(q-1)^{2}\left(c_{n-1,21,0}+c_{n-1,21,1} q+c_{n-1,21,2} q^{2}\right) & \bmod q^{3} .
\end{aligned}
$$

Extracting the coefficient of $q^{2}$ gives

$$
c_{n, 21,2}=c_{n-1,2,2}-c_{n-1,2,1}+c_{n-1,12,1}+\left(-c_{n-1,21,2}+2 c_{n-1,21,1}-c_{n-1,21,0}\right)
$$


or, since $C_{n-1,2}(q)=q \cdot C_{n-2,1}(q)$ by (3.8), we have $c_{n-1,2,2}=c_{n-1,1,1}$, so

$$
\begin{aligned}
c_{n, 21,2} & =c_{n-2,1,1}-c_{n-1,2,1}+c_{n-1,12,1}+\left(-c_{n-1,21,2}+2 c_{n-1,21,1}-c_{n-1,21,0}\right) \\
& =(-1)^{n-2}(n-1)-(-1)^{n-1}+(-1)^{n-1}(n-1)-c_{n-1,21,2}+2(-1)^{n-1}-0,
\end{aligned}
$$

substituting from (3.28) and (3.27),

$$
=-c_{n-1,21,2}+(-1)^{n-1} \text {. }
$$

We compute an alternating sum of another sequence of equalities

$$
\begin{aligned}
c_{n, 21,2}+c_{n-1,21,2} & =(-1)^{n-1} \\
(-1)\left(c_{n, 21,2}+c_{n-1,21,2}\right) & =(-1)(-1)^{n-2} \\
& \cdots \\
(-1)^{k-1}\left(c_{n-k+1,21,2}+c_{n-k, 21,2}\right) & =(-1)^{k-1}(-1)^{n-k} \\
(-1)^{k}\left(c_{n-k, 21,2}+c_{n-k-1,21,2}\right) & =(-1)^{k}(-1)^{n-k-1}
\end{aligned}
$$

Adding the equations we get

$$
c_{n, 21,2}+(-1)^{k} c_{n-k-1,21,2}=(-1)^{n-1}(k+1) \text {, or } c_{n, 21,2}+(-1)^{n-j-1} c_{j, 21,2}=(-1)^{n-1}(n-j),
$$

if we write $k=n-j-1$, so that $j=n-k-1$. Referring to section 9, the first $j$ for which $c_{j, 21,2} \neq 0$ is $j=3$, and $C_{3,21}(q)=-q+2 q^{2}-q^{3}$, so $c_{3,21,2}=2$. Substituting and rearranging,

$$
c_{n, 21,2}=(-1)^{n-1}(n-3)-(-1)^{n} c_{3,21,2}=(-1)^{n-1}((n-3)+2)=(-1)^{n-1}(n-1),
$$

and this holds for $n \geq 3$. Substituting into (5.9), we get

$$
p_{4}=c_{n-1,1,3}+(-1)^{n-2}(n-2)+2(-1)^{n-1}=c_{n-1,1,3}+(-1)^{n} n+4(-1)^{n-1} .
$$

The most straightforward approach to computing $c_{n-1,1,3}$ is through the recursion relation (3.7). Reducing the relation $\bmod q^{4}$ gives

$$
\begin{aligned}
c_{n, 1,0}+c_{n, 1,1} q+c_{n, 1,2} q^{2} & +c_{n, 1,3} q^{3} \\
(-1+2 q & \left.-q^{2}\right)\left(c_{n-1,1,0}+c_{n-1,1,1} q+c_{n-1,1,2} q^{2}+c_{n-1,1,3} q^{3}\right) \\
& +(1-q)\left(c_{n-1,0,0}+c_{n-1,0,1} q+c_{n-1,0,2} q^{2}+c_{n-1,0,3} q^{3}\right) \quad \bmod q^{4},
\end{aligned}
$$

and extracting the coefficient of $q^{3}$ gives

$$
\begin{aligned}
c_{n, 1,3} & =-c_{n-1,1,3}+2 c_{n-1,1,2}-c_{n-1,1,1}+c_{n-1,0,3}-c_{n-1,0,2} \\
& =-c_{n-1,1,3}+2 c_{n-1,1,2}+c_{n-1,0,3},
\end{aligned}
$$

since $c_{n-1,1,1}+c_{n-1,0,2}=(-1)^{n-1} n+(-1)^{n-2} n=0$ for $n \geq 4$ by (3.28) and (4.7). Making the substitution for $c_{n-1,0,3}$ from (5.3),

$$
\begin{aligned}
c_{n, 1,3}+c_{n-1,1,3} & =2(-1)^{n-2}\left(\frac{n(n-1)}{2}+1\right)+c_{n-1,0,3} \\
& =(-1)^{n-2}(n(n-1)+2)+(-1)^{n-1}(n-2)+c_{n-2,1,2} \\
& =(-1)^{n-2}(n(n-1)+2)+(-1)^{n-1}(n-2)+(-1)^{n-3}((n-1)(n-2) / 2+1) \\
& =(-1)^{n} \frac{n^{2}}{2}+(-1)^{n-1} \frac{n}{2}+(-1)^{n} 2
\end{aligned}
$$


Now generate a telescoping sum from the chain of equalities

$$
\begin{aligned}
c_{n, 1,3}+c_{n-1,1,3} & =(-1)^{n}\left[\frac{n^{2}}{2}-\frac{n}{2}+2\right] \\
(-1)\left[c_{n-1,1,3}+c_{n-2,1,3}\right] & =(-1)(-1)^{n-1}\left[\frac{(n-1)^{2}}{2}-\frac{n-1}{2}+2\right] \\
& \ldots \\
(-1)^{k}\left[c_{n-k, 1,3}+c_{n-k-1,1,3}\right] & =(-1)^{k}(-1)^{n-k}\left[\frac{(n-k)^{2}}{2}-\frac{(n-k)}{2}+2\right]
\end{aligned}
$$

Adding these equalities gives

$$
c_{n, 1,3}+(-1)^{k} c_{n-k-1,1,3}=(-1)^{n}\left[\frac{1}{2} \sum_{j=n-k}^{n} j^{2}-\frac{1}{2} \sum_{j=n-k}^{n} j+(k+1) 2\right]
$$

and, if we write $n-k-1=\ell$, so that $n-k=\ell+1$ and $k+1=n-\ell$,

$$
\begin{aligned}
& c_{n, 1,3}+(-1)^{n-\ell-1} c_{\ell, 1,3}=(-1)^{n} {\left[\frac{1}{2} \sum_{j=\ell+1}^{n} j^{2}-\frac{1}{2} \sum_{j=\ell+1}^{n} j+(n-\ell) 2\right] } \\
&=(-1)^{n}\left[\frac{1}{2} \frac{n(n+1)(2 n+1)}{6}-\frac{1}{2} \frac{\ell(\ell+1)(2 \ell+1)}{6}\right. \\
&\left.\quad-\frac{1}{2} \frac{n(n+1)}{2}+\frac{1}{2} \frac{\ell(\ell+1)}{2}+(n-\ell) 2\right]
\end{aligned}
$$

Taking $\ell=4$, so that, from section 9, $C_{4,1}(q)=-1+5 q-11 q^{2}+16 q^{3}-\cdots$ and $c_{4,1,3}=16$, we evaluate

$$
\begin{aligned}
c_{n, 1,3}-(-1)^{n}(16) & =(-1)^{n}\left[\frac{1}{2} \frac{2 n^{3}}{6}+\frac{1}{2} \frac{3 n^{2}}{6}+\frac{1}{2} \frac{n}{6}-\frac{1}{2} \frac{4 \cdot 5 \cdot 9}{6}\right. \\
& \left.\quad-\frac{1}{2} \frac{n^{2}}{2}-\frac{1}{2} \frac{n}{2}+\frac{1}{2} \frac{4 \cdot 5}{2}+2(n-4)\right] \\
& =(-1)^{n}\left[\frac{n^{3}}{6}-\frac{n}{6}-10+2 n-8\right] \\
& =(-1)^{n}\left[\frac{n(n-1)(n+1)}{6}+2 n-18\right]
\end{aligned}
$$

Therefore,

$$
c_{n, 1,3}=(-1)^{n}\left[\frac{n(n-1)(n+1)}{6}+2 n-18+16\right]=(-1)^{n}\left[\frac{n(n-1)(n+1)}{6}+2 n-2\right],
$$

a formula valid for $n \geq 4$, but failing for $n=3$, so

$$
\begin{aligned}
\lambda_{-n+3}=p_{4} & =c_{n-1,1,3}+(-1)^{n} n+4(-1)^{n-1} \\
& =(-1)^{n-1}\left[\frac{n(n-1)(n-2)}{6}+2(n-1)-2\right]-(-1)^{n-1} n+4(-1)^{n-1} \\
& =(-1)^{n-1}(n(n-1)(n-2) / 6+n),
\end{aligned}
$$

which is consequently valid for $n \geq 5$. This ends the proof. 


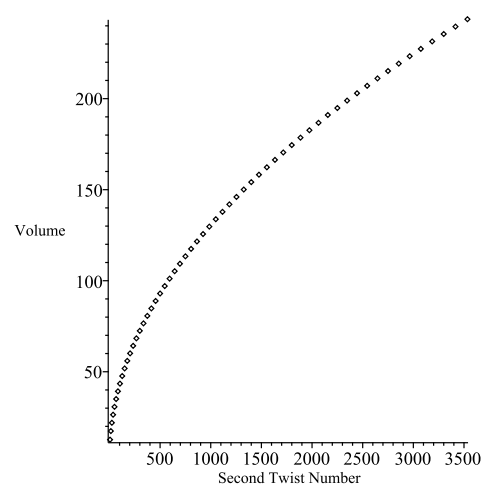

Figure 8: Second twist number versus volume

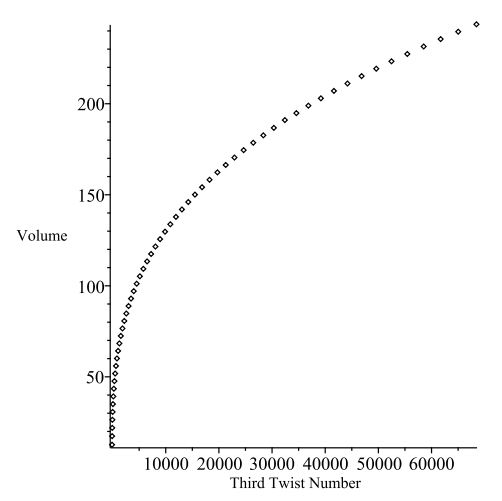

Figure 9: Third twist number versus volume

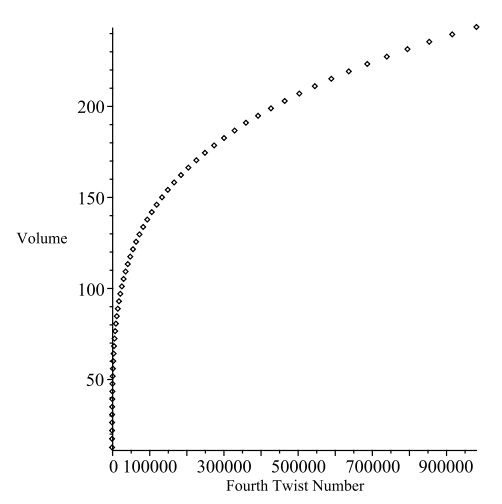

Figure 10: Fourth twist number versus volume

\section{From the Jones Polynomial to Khovanov homology}

In this section we amplify Theorem 2.6, at least the first part of it.

Theorem 3.5. For a weaving knot $W(2 k+1, n)$ the non-vanishing Khovanov homology $\mathcal{H}^{i, j}(W(2 k+1, n))$ lies on the lines

$$
j=2 i \pm 1 .
$$


For a weaving knot $W(2 k, n)$ the non-vanishing Khovanov homology $\mathcal{H}^{i, j}(W(2 k, n))$ lies on the lines

$$
j=2 i+n-1 \pm 1
$$

We have the following definition of the bi-graded Euler characteristic associated to Khovanov homology.

$$
K h(L)(t, Q) \stackrel{\text { def }}{=} \sum t^{i} Q^{j} \operatorname{dim} \mathcal{H}^{i, j}(L)
$$

Theorem 6.1 (Theorem 1.1, [9]). For an oriented link L, the graded Euler characteristic

$$
\sum_{i, j \in \mathbf{Z}}(-1)^{i} Q^{j} \operatorname{dim} \mathcal{H}^{i, j}(L)
$$

of the Khovanov invariant $\mathcal{H}(L)$ is equal to $\left(Q^{-1}+Q\right)$ times the Jones polynomial $V_{L}\left(Q^{2}\right)$ of $L$.

In terms of the associated polynomial $K h(L)$,

$$
K h(L)(-1, Q)=\left(Q^{-1}+Q\right) V_{L}\left(Q^{2}\right)
$$

Theorem 6.2 (Compare Theorem 1.4 and subsequent remarks from [9]). For an alternating knot $L$, its Khovanov invariants $\mathcal{H}^{i, j}(L)$ of degree difference $(1,4)$ are paired except in the 0th cohomology group.

This fact may be expressed in terms of the polynomial $K h(L)$, as follows. There is another polynomial $K h^{\prime}(L)$ in one variable and an equality

$$
K h(L)(t, Q)=Q^{-\sigma(L)}\left\{\left(Q^{-1}+Q\right)+\left(Q^{-1}+t Q^{2} \cdot Q\right) \cdot K h^{\prime}(L)\left(t Q^{2}\right)\right\}
$$

When we combine theorems 6.1 and 6.2, we find that the bi-graded Euler characteristic and the Jones polynomial of an alternating link determine one another. Obviously, the equality (6.1) shows that one knows $V_{L}$ if one knows $K h(t, Q)$.

To obtain $K h(t, Q)$ from $V_{L}\left(Q^{2}\right)$ requires a certain amount of manipulation. Implementing these manipulations in Maple and Mathematica is an important step in our experiments. Setting $t=-1$ in (6.2) and combining with equation (6.1), one has

$$
\left(Q^{-1}+Q\right) \cdot V_{L}\left(Q^{2}\right)=Q^{-\sigma(L)}\left\{\left(Q^{-1}+Q\right)+\left(Q^{-1}-Q^{3}\right) \cdot K h^{\prime}(L)\left(-Q^{2}\right)\right\} .
$$

Consequently,

$$
\begin{aligned}
V_{L}\left(Q^{2}\right) & =Q^{-\sigma(L)}\left\{1+\frac{\left(Q^{-1}-Q^{3}\right)}{\left(Q^{-1}+Q\right)} \cdot K h^{\prime}(L)\left(-Q^{2}\right)\right\} \\
& =Q^{-\sigma(L)}\left\{1+\left(1-Q^{2}\right) \cdot K h^{\prime}(L)\left(-Q^{2}\right)\right\} .
\end{aligned}
$$

Furthermore,

$$
Q^{\sigma(L)} \cdot V_{L}\left(Q^{2}\right)-1=\left(1-Q^{2}\right) \cdot K h^{\prime}(L)\left(-Q^{2}\right)
$$


or

$$
K h^{\prime}(L)\left(-Q^{2}\right)=\left(1-Q^{2}\right)^{-1} \cdot\left(Q^{\sigma(L)} \cdot V_{L}\left(Q^{2}\right)-1\right) .
$$

Replacing $Q^{2}$ in the last equation by $-t Q^{2}$ is the last step to obtain $K h^{\prime}(L)$ from the Jones polynomial. Within a computer algebra system, one must first replace $Q^{2}$ by $-X$ and then replace $X$ by $t Q^{2}$. Once one has $K h^{\prime}(L)\left(t Q^{2}\right)$, one obtains $K h(t, Q)$ directly from equation (6.2).

Example 6.3. We have computed $V_{W(3,2)}(t)=t^{-2}-t^{-1}+1-t+t^{2}$ in example 4.4, so

$$
\begin{aligned}
K h^{\prime}(W(3,2))\left(-Q^{2}\right) & =\left(1-Q^{2}\right)^{-1} \cdot\left(Q^{0} \cdot\left(Q^{-4}-Q^{-2}-Q^{2}+Q^{4}\right)\right) \\
& =\left(1-Q^{2}\right)^{-1} \cdot\left(\left(1-Q^{2}\right) \cdot\left(Q^{-4}-Q^{2}\right)\right) \\
& =Q^{-4}-Q^{2} .
\end{aligned}
$$

It follows that $K h^{\prime}(W(3,2))\left(t Q^{2}\right)=t^{-2} Q^{-4}+t Q^{2}$, and

$$
\begin{aligned}
K h(W(3,2))(t, Q)=\left(Q+Q^{-1}\right)+\left(Q^{-1}+t Q^{3}\right)\left(t^{-2} Q^{-4}+t Q^{2}\right) \\
\\
=t^{-2} Q^{-5}+t^{-1} Q^{-1}+Q^{-1}+Q+t Q+t^{2} Q^{5} .
\end{aligned}
$$

\section{$7 \quad$ Khovanov homology examples}

Once one has the Khovanov polynomial one can make a plot of the Khovanov homology in an $(i, j)$-plane as in this example. The Betti number $\operatorname{dim} \mathcal{H}^{i, j}(W(3,11))$ is plotted at the point with coordinates $(i, j)$. Clearly, as $n$ gets larger, it is going to be harder to make sense

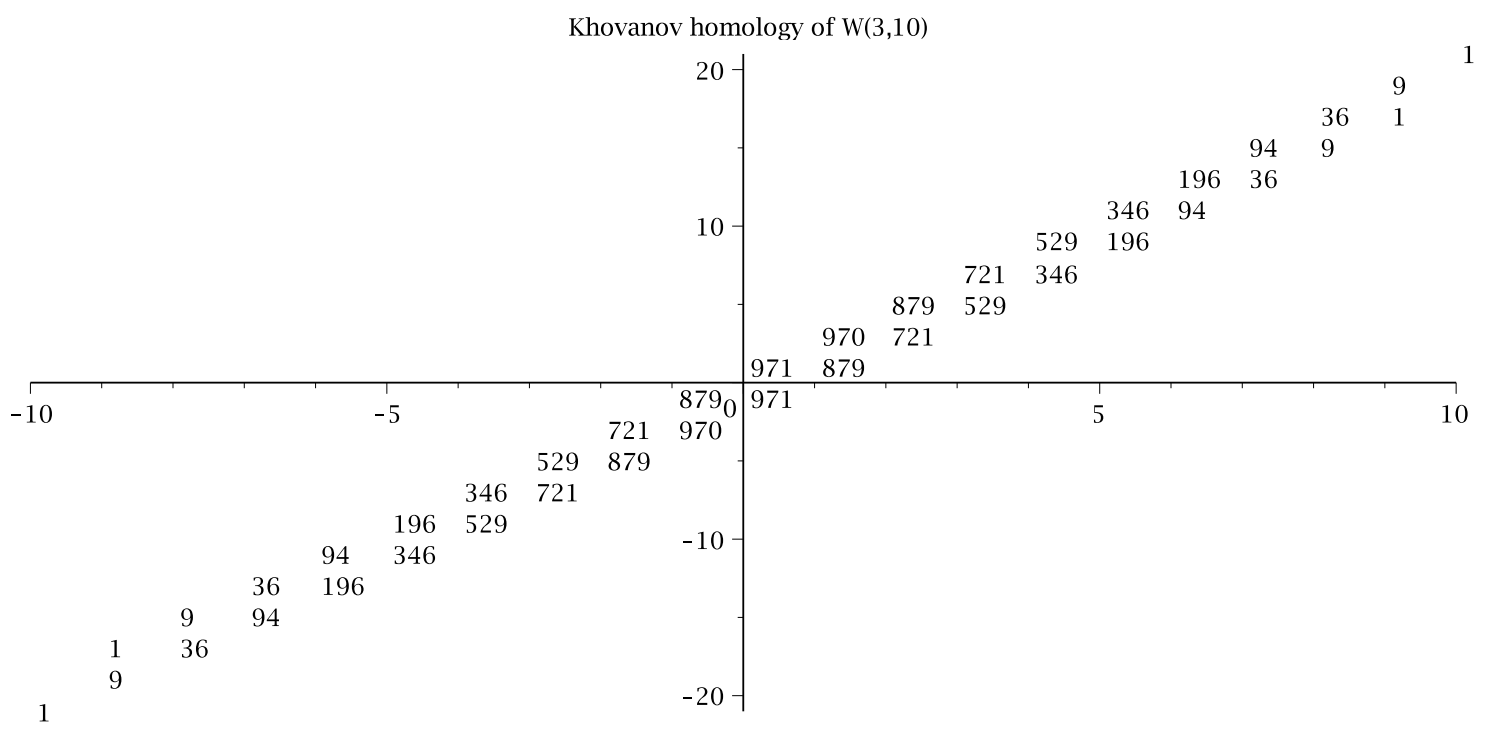

Figure 11: Khovanov homology of $W(3,10)$

of such plots. Notice that the "knight move" $(1,4)$-periodicity of the Khovanov homology for these knots essentially makes the information on one of the lines $j-2 i= \pm 1$ redundant. 
Table 2: Integral Khovanov homology of $W(3,4)$

\begin{tabular}{||c|c|c|c|c|c|c|c|c|c||}
\hline \hline & -4 & -3 & -2 & -1 & 0 & 1 & 2 & 3 & 4 \\
\hline 9 & & & & & & & & & 1 \\
\hline 7 & & & & & & & & 3 & $1_{2}$ \\
\hline 5 & & & & & & & 3 & $1,3_{2}$ & \\
\hline 3 & & & & & & 4 & $3,3_{2}$ & & \\
\hline 1 & & & & & 5 & $3,4_{2}$ & & & \\
\hline-1 & & & & 3 & $5,4_{2}$ & & & & \\
\hline-3 & & & 3 & $4,3_{2}$ & & & & & \\
\hline-5 & & 1 & $3,3_{2}$ & & & & & & \\
\hline-7 & & $3,1_{2}$ & & & & & & & \\
\hline-9 & 1 & & & & & & & & \\
\hline \hline
\end{tabular}

Before we continue to explore Khovanov homology with rational coefficients, we observe we can also compute the integral Khovanov homology. By Corollary 5 of [17] there is only torsion of order 2 in the integral Khovanov homology. Even better, there are rules for calculating the number of $\mathbf{Z} / 2 \mathbf{Z}$-summands present. We have already noted how rational Khovanov homology spaces are related by so-called "knight moves." Except when one lands or starts in a space $\mathcal{H}^{0, *}(W(3, n))$, a move from $\mathcal{H}^{i, j}(W(3, n))$ to $\mathcal{H}^{i+1, j+4}(W(3, n))$ is a move from one space into another space of the same dimension. If one reduces the dimensions of the spaces $\mathcal{H}^{0, *}(W(3, n))$ by one, then this phenomenon persists without qualification on the bidegrees. Having made this adjustment, Shumakovitch [17, 1.G Definitions] provides the following rules for computing the integral Khovanov homology: If $\operatorname{dim} \mathcal{H}^{i, 2 i+1}(W(3, n))=$ $a \neq 0$, then $\mathcal{H}^{i, 2 i+1}(W(3, n) ; \mathbf{Z})$ is torsion-free of rank $a$. Moreover, for every non-zero pair linked by a knight move, $\mathcal{H}^{i-1,2 i-3}(W(3, n) ; \mathbf{Z})$ and $\mathcal{H}^{i, 2 i+1}(W(3, n) ; \mathbf{Z})$ have the same rank, but groups along the line $j-2 i=-1$ may have torsion. In fact, the two-torsion part of $\mathcal{H}^{i, 2 i-1}(W(3, n) \mathbf{Z})$ is an abelian 2-group $(\mathbf{Z} / 2 \mathbf{Z})^{a}$. The table 2 shows the rules in operation for the knot $W(3,4)$, also identified as $8 \_18$ in standard knot tables. Integer entries along the line $j-2 i=1$ indicate the ranks of free abelian groups and an entry $r, a_{2}$ along the line $j-2 i=-1$ indicates a free abelian group of rank $r$ summed with a 2 -group $(\mathbf{Z} / 2 \mathbf{Z})^{a}$.

Returning to rational Khovanov homology, we take advantage of the "knight move" periodicity and simplify by recording the Betti numbers from only along the line $j-2 i=1$. In order to study the asymptotic behavior of Khovanov homology we have to normalize the data. This is done by computing the total rank of the Khovanov homology along the line and dividing each Betti number by the total rank. We obtain normalized Betti numbers that sum to one.

This raises the possibility of approximating the distribution of normalized Betti numbers by a probability distribution. For our baseline experiments we choose to use the normal $N\left(\mu, \sigma^{2}\right)$ probability density function

$$
f_{\mu, \sigma^{2}}(x)=\frac{1}{\sigma \sqrt{2 \pi}} \exp \left(-\frac{(x-\mu)^{2}}{2 \sigma^{2}}\right)
$$


Fit a quadratic function $q_{n}(x)=-\left(\alpha x^{2}-\beta x+\delta\right)$ to the logarithms of the normalized Khovanov dimensions along the line $j=2 i+1$ and exponentiate the quadratic function. Since the total of the normalized dimensions is 1 , we normalize the exponential, obtaining

$$
\rho_{n}(x)=A_{n} e^{q_{n}(x)} \quad \text { satisfying } \quad \int_{-\infty}^{\infty} \rho_{n}(x) d x=1 .
$$

To obtain a formula for $A_{n}$, complete the square

$$
q_{n}(x)=-\alpha \cdot(x-(\beta / 2 \alpha))^{2}+\left(\left(\beta^{2} / 4 \alpha\right)-\delta\right) .
$$

Then consider

$$
\begin{aligned}
1 & =A_{n} \int_{-\infty}^{\infty} \exp q_{n}(x) d x \\
& =A_{n} \cdot \int_{-\infty}^{\infty} \exp \left(\left(\beta^{2} / 4 \alpha\right)-\delta\right) \cdot \exp \left(-\alpha \cdot(x-(\beta / 2 \alpha))^{2}\right) d x \\
& =A_{n} \cdot \exp \left(\left(\beta^{2} /(4 \alpha)-\delta\right) \cdot \int_{-\infty}^{\infty} \exp \left(-\alpha \cdot(x-(\beta / 2 \alpha))^{2}\right) d x\right. \\
& =A_{n} \cdot \exp \left(\left(\beta^{2} / 4 \alpha\right)-\delta\right) \cdot \sqrt{\pi / \alpha}
\end{aligned}
$$

Thus, the expression for $A_{n}$ is

$$
A_{n}=\exp \left(-\left(\left(\beta^{2} / 4 \alpha\right)-\delta\right)\right) \cdot \sqrt{\alpha / \pi}
$$

Equate the expressions

$$
\rho_{n}(x)=\frac{1}{\sigma_{n} \sqrt{2 \pi}} \exp \left(-\frac{\left(x-\mu_{n}\right)^{2}}{2 \sigma_{n}^{2}}\right) \quad \text { and } \quad \rho_{n}(x)=A_{n} \exp \left(q_{n}(x)\right),
$$

and observe $\mu_{n}=\beta / 2 \alpha$ by equating the two expressions for the location of the local maximum of $\rho_{n}(x)$. Then the efficient way to the parameter $\sigma_{n}$ is to solve the equation

$\frac{1}{\sigma_{n} \sqrt{2 \pi}}=\rho_{n}(\beta / 2 \alpha)=A_{n} \exp \left(q_{n}(\beta / 2 \alpha)\right)=\exp \left(-\left(\left(\beta^{2} / 4 \alpha\right)-\delta\right)\right) \cdot \sqrt{\alpha / \pi} \cdot \exp \left(\left(\beta^{2} / 4 \alpha\right)-\delta\right)$,

obtaining $\sigma_{n}=1 / \sqrt{2 \alpha}$.

Working this out for $W(3,10)$, and carrying only 3 decimal places, the raw dimensions are

\begin{tabular}{c|c|c|c|c|c|c|c|c|c|c}
$i$ & -9 & -8 & -7 & -6 & -5 & -4 & -3 & -2 & -1 & 0 \\
$\operatorname{dim}$ & 1 & 9 & 36 & 94 & 196 & 346 & 529 & 721 & 879 & 970 \\
$i$ & 1 & 2 & 3 & 4 & 5 & 6 & 7 & 8 & 9 & 10 \\
$\operatorname{dim}$ & 971 & 879 & 721 & 529 & 346 & 196 & 94 & 36 & 9 & 1
\end{tabular}

and, to three significant digits, the logarithms of the normalized dimensions are

$i \quad$\begin{tabular}{c|c|c|c|c|c|c|c|c|c}
-9 & -8 & -7 & -6 & -5 & -4 & -3 & -2 & -1 & 0 \\
-17.9 & -15.7 & -14.3 & -13.3 & -12.6 & -12.0 & -11.6 & -11.3 & -11.1 & -11.0 \\
1 & 2 & 3 & 4 & 5 & 6 & 7 & 8 & 9 & 10 \\
-11.0 & -11.1 & -11.3 & -11.6 & -12.0 & -12.6 & -13.3 & -14.3 & -15.7 & -17.9
\end{tabular}


Fitting a quadratic to this information, we get

$$
q_{10}(x)=-10.7+0.0720 x-0.0720 x^{2}, \quad \alpha=\beta=0.0720, \quad \delta=10.7 .
$$

To three significant digits $\mu_{10}=0.500$ and $\sigma_{10}=2.64$.

By the symmetry of Khovanov homology, the mean $\mu_{n}$ approaches $1 / 2$ rapidly, so this parameter is of little interest. On the other hand, relating the parameter $\sigma_{n}$ to some geometric quantity, say, some hyperbolic invariant of the complement of the link, is a very interesting problem.

For $W(3,10)$, the density function is

$$
\begin{aligned}
& \rho_{10}(x)=11686.8431618280538 \sqrt{\pi^{-1}} \\
& \cdot \exp \left(-10.7018780565714309+0.0716848579220777243 x-0.0716848579220778631 x^{2}\right)
\end{aligned}
$$

When placed into standard form, $\mu_{10}=0.5000054030$ and $\sigma_{10}=2.640882970$. Figure 12 compares the plot of the density function $\rho_{10}$ with a point plot of normalized dimensions.

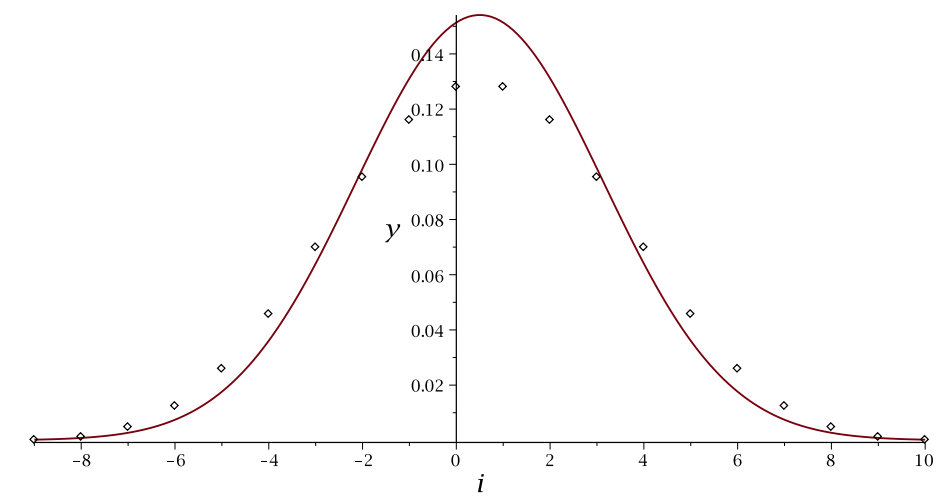

Figure 12: normalized homology of $W(3,10)$ compared with density function

For the knot $W(3,11)$ the expression for the density function is

$$
\begin{aligned}
& \rho_{11}(x)=29676.8676257830375 \sqrt{\pi^{-1}} \\
& \cdot \exp \left(-11.6724860231789886+0.0661625395821569817 x-0.0661623073574252735 x^{2}\right)
\end{aligned}
$$

When placed into standard form, $\mu_{11}=0.5000017550$ and $\sigma_{11}=2.749031276$. Figure 13 compares the plot of the density function $\rho_{11}$ with a point plot of normalized dimensions. For $W(3,22)$, the density function is

$$
\begin{aligned}
& \rho_{22}(x)=833596689.149608016 \sqrt{\pi^{-1}} \\
& \cdot \exp \left(-22.2219365040983057+0.0353061029354434300 x-0.0353061029347388616 x^{2}\right)
\end{aligned}
$$

When placed into standard form, $\mu_{22}=0.500000000$ and $\sigma_{22}=3.763224354$. Figure 14 compares the plot of the density function $\rho_{22}$ with a point plot of normalized dimensions. 


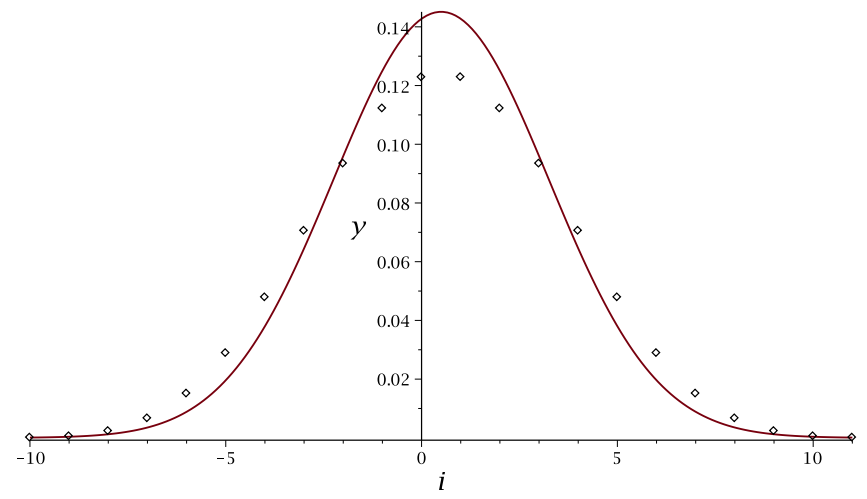

Figure 13: normalized homology of $W(3,11)$ compared with density function

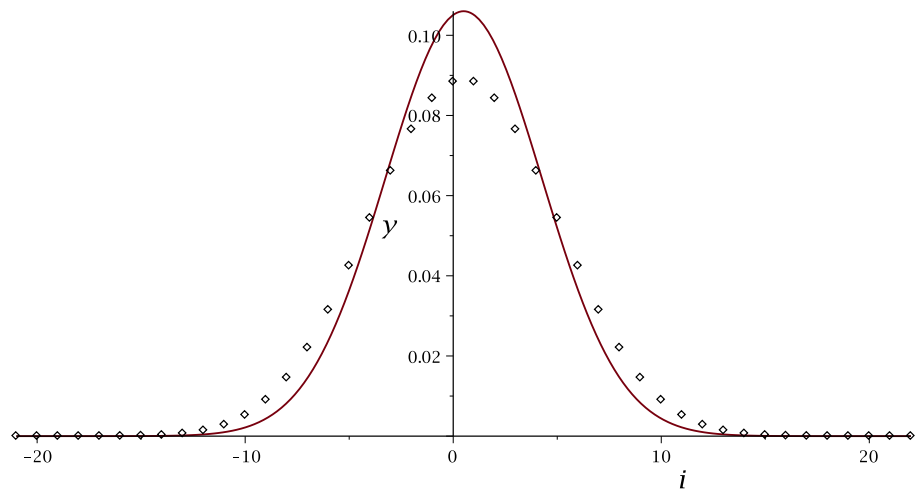

Figure 14: normalized homology of $W(3,22)$ compared with density function

For $W(3,23)$, the density function is

$$
\begin{aligned}
& \rho_{23}(x)=2113964949.23002362 \sqrt{\pi^{-1}} \\
& \cdot \exp \left(-23.1731352596503442+0.0338545815354610105 x-0.0338545815348441914 x^{2}\right)
\end{aligned}
$$

When placed into standard form, $\mu_{23}=0.5000000000$ and $\sigma_{23}=3.843052143$. Figure 15 compares the plot of the density function $\rho_{23}$ with a point plot of normalized dimensions. Maple worksheets and, later, Mathematica notebooks will be available at URL [11] prepared by the second-named author.

\section{Data Tables}

This section contains tables of data generated using Maple to implement some of the results of earlier sections. The first table collects data for weaving knots $W(3, n)$ with $n \equiv 1 \bmod 3$; the second table does the same for weaving knots $W(3, n)$ with $n \equiv 2 \bmod 3$. In each table the first column lists the value of $n$; the second column lists the total dimension of the Khovanov homology lying along the line $j=2 i+1$; and the third column lists the dimension of the vector space $\mathcal{H}^{0,1}(W(3, n))$. Columns four and five display measures of the deviation of the proposed normal distributions from the actual distribution of normalized dimensions. 


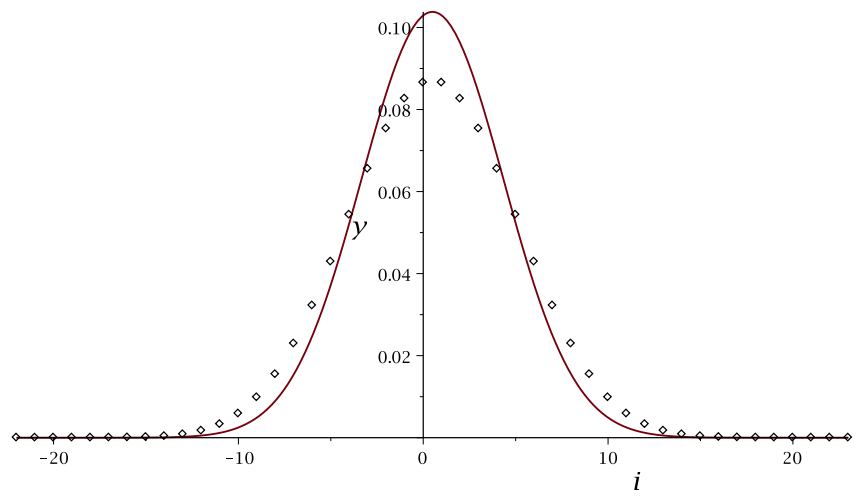

Figure 15: normalized homology of $W(3,23)$ compared with density function

In section 7 we have approximated a distribution of normalized Khovanov dimenstions by a standard normal distribution, and we have displayed graphics comparing an actual distribution with its approximation. To quantify those visual impressions, we compute and tabulate an $L^{1}$ - and an $L^{2}$-deviation. Let

$$
\text { Total dimension }=\sum_{i=-2 n}^{2 n+1} \operatorname{dim} \mathcal{H}^{i, 2 i+1}(W(3, n)) .
$$

For the $L^{2}$-comparison, we compute

$$
\left(\sum_{i=-2 n}^{2 n+1}\left(\rho_{n}(i)-\frac{\operatorname{dim} \mathcal{H}^{i, 2 i+1}(W(3, n))}{\text { Total dimension }}\right)^{2}\right)^{1 / 2}
$$

For the $L^{1}$-comparison, we compute

$$
\sum_{i=-2 n}^{2 n+1}\left|\rho_{n}(i)-\frac{\operatorname{dim} \mathcal{H}^{i, 2 i+1}(W(3, n))}{\text { Total dimension }}\right|
$$

The $L^{2}$ comparisons appear to tend to 0 , whereas the $L^{1}$ comparisons appear to be growing slowly. 
Table 3: Data for $W(3, n)$ with $n \equiv 1 \bmod 3$

\begin{tabular}{|c|c|c|c|c|c|}
\hline$n$ & Total dimension & $\operatorname{dim} \mathcal{H}^{0,1}$ & $\sigma$ & $L^{2}$-comparison & $L^{1}$ comparison \\
\hline 10 & 7563 & 970 & 2.64088 & 0.040510 & 0.134828 \\
\hline 13 & 135721 & 15418 & 2.95616 & 0.041133 & 0.150599 \\
\hline 16 & 2435423 & 250828 & 3.24564 & 0.040792 & 0.155995 \\
\hline 19 & 43701901 & 4146351 & 3.51339 & 0.040145 & 0.161336 \\
\hline 22 & 784198803 & 69337015 & 3.76322 & 0.039413 & 0.165763 \\
\hline 25 & 14071876561 & 1169613435 & 3.99810 & 0.038678 & 0.167576 \\
\hline 28 & 252509579303 & 19864129051 & 4.22032 & 0.037971 & 0.167790 \\
\hline 31 & 4531100550901 & 339205938364 & 4.43167 & 0.037303 & 0.170736 \\
\hline 34 & 81307300336923 & 5818326037345 & 4.63358 & 0.036676 & 0.172392 \\
\hline 37 & 1459000305513721 & 100173472277125 & 4.82719 & 0.036089 & 0.173119 \\
\hline 40 & 26180698198910063 & 1730135731194046 & 5.01342 & 0.035541 & 0.173178 \\
\hline 43 & 469793567274867421 & 29963026081609060 & 5.19306 & 0.035028 & 0.173812 \\
\hline 46 & 8430103512748703523 & 520131503664409798 & 5.36674 & 0.034546 & 0.175052 \\
\hline 49 & $1.51272 \cdot 10^{20}$ & $9.04765 \cdot 10^{18}$ & 5.53502 & 0.034093 & 0.175779 \\
\hline 52 & $2.71447 \cdot 10^{21}$ & $1.57670 \cdot 10^{20}$ & 5.69838 & 0.033667 & 0.176100 \\
\hline 55 & $4.87091 \cdot 10^{22}$ & $2.75210 \cdot 10^{21}$ & 5.85721 & 0.033265 & 0.176098 \\
\hline 58 & $8.74050 \cdot 10^{23}$ & $4.81071 \cdot 10^{22}$ & 6.01187 & 0.032885 & 0.175898 \\
\hline 61 & $1.56842 \cdot 10^{25}$ & $8.42017 \cdot 10^{23}$ & 6.16267 & 0.032524 & 0.176778 \\
\hline 64 & $2.81441 \cdot 10^{26}$ & $1.47552 \cdot 10^{25}$ & 6.30989 & 0.032182 & 0.177369 \\
\hline 67 & $5.05026 \cdot 10^{27}$ & $2.58843 \cdot 10^{26}$ & 6.45376 & 0.031857 & 0.177716 \\
\hline 70 & $9.06233 \cdot 10^{28}$ & $4.54520 \cdot 10^{27}$ & 6.59451 & 0.031547 & 0.177859 \\
\hline 73 & $1.62617 \cdot 10^{30}$ & $7.98842 \cdot 10^{28}$ & 6.73233 & 0.031251 & 0.177831 \\
\hline 76 & $2.91804 \cdot 10^{31}$ & $1.40517 \cdot 10^{30}$ & 6.86740 & 0.030968 & 0.177657 \\
\hline 79 & $5.23621 \cdot 10^{32}$ & $2.47359 \cdot 10^{31}$ & 6.99986 & 0.030697 & 0.177995 \\
\hline 82 & $9.39600 \cdot 10^{33}$ & $4.35747 \cdot 10^{32}$ & 7.12988 & 0.030437 & 0.178445 \\
\hline 85 & $1.68604 \cdot 10^{35}$ & $7.68116 \cdot 10^{33}$ & 7.25757 & 0.030188 & 0.178746 \\
\hline 88 & $3.02548 \cdot 10^{36}$ & $1.35483 \cdot 10^{35}$ & 7.38305 & 0.029948 & 0.178918 \\
\hline 91 & $5.42901 \cdot 10^{37}$ & $2.39106 \cdot 10^{36}$ & 7.50645 & 0.029718 & 0.178976 \\
\hline 94 & $9.74196 \cdot 10^{38}$ & $4.22211 \cdot 10^{37}$ & 7.62786 & 0.029496 & 0.178935 \\
\hline 97 & $1.74812 \cdot 10^{40}$ & $7.45910 \cdot 10^{38}$ & 7.74736 & 0.029282 & 0.178807 \\
\hline 100 & $3.13688 \cdot 10^{41}$ & $1.31840 \cdot 10^{40}$ & 7.86506 & 0.029075 & 0.178890 \\
\hline 121 & $1.87923 \cdot 10^{50}$ & $7.18477 \cdot 10^{48}$ & 8.64424 & 0.027805 & 0.179577 \\
\hline 142 & $1.12580 \cdot 10^{59}$ & $3.97500 \cdot 10^{57}$ & 9.35886 & 0.026769 & 0.180247 \\
\hline 163 & $6.74436 \cdot 10^{67}$ & $2.22337 \cdot 10^{66}$ & 10.0227 & 0.025900 & 0.180596 \\
\hline 184 & $4.04037 \cdot 10^{76}$ & $1.25398 \cdot 10^{75}$ & 10.6453 & 0.025156 & 0.180629 \\
\hline 205 & $2.42049 \cdot 10^{85}$ & $7.11854 \cdot 10^{83}$ & 11.2334 & 0.024508 & 0.180907 \\
\hline 247 & $8.68689 \cdot 10^{102}$ & $2.32816 \cdot 10^{101}$ & 12.3258 & 0.023423 & 0.181027 \\
\hline 289 & $3.11764 \cdot 10^{120}$ & $7.72623 \cdot 10^{118}$ & 13.3289 & 0.022542 & 0.181268 \\
\hline
\end{tabular}


Table 4: Data for $W(3, n)$ with $n \equiv 2 \bmod 3$

\begin{tabular}{|c|c|c|c|c|c|}
\hline$n$ & Total dimension & $\operatorname{dim} \mathcal{H}^{0,1}$ & $\sigma$ & $L^{2}$-comparison & $L^{1}$ comparisc \\
\hline 11 & 19801 & 2431 & 2.74903 & 0.040906 & 0.141925 \\
\hline 14 & 355323 & 38983 & 3.05533 & 0.041079 & 0.153170 \\
\hline 17 & 6376021 & 637993 & 3.33710 & 0.040595 & 0.156595 \\
\hline 20 & 114413063 & 10591254 & 3.59850 & 0.039905 & 0.163190 \\
\hline 23 & 2053059121 & 177671734 & 3.84305 & 0.039166 & 0.166596 \\
\hline 26 & 36840651123 & 3004390818 & 4.07348 & 0.038438 & 0.167789 \\
\hline 29 & 661078661101 & 51124396786 & 4.29190 & 0.037744 & 0.168941 \\
\hline 32 & 11862575248703 & 874400336044 & 4.49997 & 0.037089 & 0.171411 \\
\hline 35 & 212865275815561 & 15018149469823 & 4.69899 & 0.036476 & 0.172723 \\
\hline 38 & 3819712389431403 & 258853011125599 & 4.89004 & 0.035903 & 0.173203 \\
\hline 41 & 68541957733949701 & 4474997964407374 & 5.07400 & 0.035366 & 0.173083 \\
\hline 44 & 1229935526821663223 & 77563025486587315 & 5.25158 & 0.034864 & 0.174290 \\
\hline 47 & 22070297525055988321 & 1347390412214087833 & 5.42341 & 0.034392 & 0.175346 \\
\hline 50 & $3.96035 \cdot 10^{20}$ & $2.34525 \cdot 10^{19}$ & 5.59000 & 0.033949 & 0.175926 \\
\hline 53 & $7.10657 \cdot 10^{21}$ & $4.08927 \cdot 10^{20}$ & 5.75181 & 0.033531 & 0.176131 \\
\hline 56 & $1.27522 \cdot 10^{23}$ & $7.14133 \cdot 10^{21}$ & 5.90921 & 0.033136 & 0.176037 \\
\hline 59 & $2.28829 \cdot 10^{24}$ & $1.24888 \cdot 10^{23}$ & 6.06255 & 0.032763 & 0.176227 \\
\hline 62 & $4.10617 \cdot 10^{25}$ & $2.18679 \cdot 10^{24}$ & 6.21213 & 0.032408 & 0.177005 \\
\hline 65 & $7.36823 \cdot 10^{26}$ & $3.83347 \cdot 10^{25}$ & 6.35821 & 0.032072 & 0.177510 \\
\hline 68 & $1.32218 \cdot 10^{28}$ & $6.72713 \cdot 10^{26}$ & 6.50102 & 0.031752 & 0.177785 \\
\hline 71 & $2.37255 \cdot 10^{29}$ & $1.18163 \cdot 10^{28}$ & 6.64077 & 0.031446 & 0.177867 \\
\hline 74 & $4.25736 \cdot 10^{30}$ & $2.07736 \cdot 10^{29}$ & 6.77765 & 0.031155 & 0.177787 \\
\hline 77 & $7.63953 \cdot 10^{31}$ & $3.65504 \cdot 10^{30}$ & 6.91183 & 0.030876 & 0.177602 \\
\hline 80 & $1.37086 \cdot 10^{33}$ & $6.43571 \cdot 10^{31}$ & 7.04347 & 0.030609 & 0.178163 \\
\hline 83 & $2.45990 \cdot 10^{34}$ & $1.13397 \cdot 10^{33}$ & 7.17269 & 0.030353 & 0.178561 \\
\hline 86 & $4.41412 \cdot 10^{35}$ & $1.99933 \cdot 10^{34}$ & 7.29963 & 0.030107 & 0.178817 \\
\hline 89 & $7.92082 \cdot 10^{36}$ & $3.52717 \cdot 10^{35}$ & 7.42441 & 0.029871 & 0.178949 \\
\hline 92 & $1.42133 \cdot 10^{38}$ & $6.22605 \cdot 10^{36}$ & 7.54714 & 0.029643 & 0.178972 \\
\hline 95 & $2.55048 \cdot 10^{39}$ & $1.09958 \cdot 10^{38}$ & 7.66790 & 0.029424 & 0.178901 \\
\hline 98 & $4.57665 \cdot 10^{40}$ & $1.94290 \cdot 10^{39}$ & 7.78679 & 0.029212 & 0.178747 \\
\hline 119 & $2.74175 \cdot 10^{49}$ & $1.05696 \cdot 10^{48}$ & 8.57308 & 0.027914 & 0.179650 \\
\hline 140 & $1.64251 \cdot 10^{58}$ & $5.84051 \cdot 10^{56}$ & 9.29316 & 0.026859 & 0.180257 \\
\hline 161 & $9.83989 \cdot 10^{66}$ & $3.26385 \cdot 10^{65}$ & 9.96138 & 0.025977 & 0.180552 \\
\hline 182 & $5.89483 \cdot 10^{75}$ & $1.83951 \cdot 10^{74}$ & 10.5875 & 0.025223 & 0.180539 \\
\hline 203 & $3.53144 \cdot 10^{84}$ & $1.04367 \cdot 10^{83}$ & 11.1787 & 0.024566 & 0.180926 \\
\hline 245 & $1.26740 \cdot 10^{102}$ & $3.41053 \cdot 10^{100}$ & 12.2759 & 0.023469 & 0.181064 \\
\hline 287 & $4.54858 \cdot 10^{119}$ & $1.13115 \cdot 10^{118}$ & 13.2829 & 0.022580 & 0.181221 \\
\hline 329 & $1.63244 \cdot 10^{137}$ & $3.79224 \cdot 10^{135}$ & 14.2187 & 0.021838 & 0.181399 \\
\hline
\end{tabular}




\section{More on Polynomials}

First we collect basic values of polynomials $C_{n, *}(q)$, some of which are referred to in sections 3. 4, and 5. Recall the initilization values:

$$
\begin{aligned}
& C_{1,0}(q)=0, C_{1,1}(q)=-(q-1), C_{1,2}(q)=0, C_{1,12}(q)=1, C_{1,21}(q)=0, \text { and } C_{1,121}(q)=0 \text {. } \\
& C_{2,0}(q)=q(q-1)^{2}=q-2 q^{2}+q^{3} \quad C_{3,0}(q)=-q+4 q^{2}-5 q^{3}+4 q^{4}-q^{5} \\
& C_{2,1}(q)=(q-1)^{3}=-1+3 q-3 q^{2}+q^{3} \quad C_{3,1}(q)=1-4 q+7 q^{2}-7 q^{3}+4 q^{4}-q^{5} \\
& C_{2,2}(q)=-q(q-1)=q-q^{2} \quad C_{3,2}(q)=-q+3 q^{2}-3 q^{3}+q^{4} \\
& C_{2,12}(q)=-(q-1)^{2}=-1+2 q-q^{2} \quad C_{3,12}(q)=1-3 q+4 q^{2}-3 q^{3}+q^{4} \\
& C_{2,21}(q)=q \quad C_{3,21}(q)=-q+2 q^{2}-q^{3} \\
& \begin{aligned}
C_{4,0}(q) & =q-5 q^{2}+10 q^{3}-12 q^{4}+10 q^{5}-5 q^{6}+q^{7} \\
C_{4,1}(q) & =-1+5 q-11 q^{2}+16 q^{3}-16 q^{4}+11 q^{5}-5 q^{6}+q^{7} \\
C_{4,2}(q) & =q-4 q^{2}+7 q^{3}-7 q^{4}+4 q^{5}-q^{6} \\
C_{4,12}(q) & =-1+4 q-7 q^{2}+9 q^{3}-7 q^{4}+4 q^{5}-q^{6} \\
C_{4,21}(q) & =q-3 q^{2}+4 q^{3}-3 q^{4}+q^{5}
\end{aligned}
\end{aligned}
$$

Table 5: Alexander polynomials for $W(3, n)$

\begin{tabular}{|c|c|}
\hline$n$ & $\Delta_{W(3, n)}(t)$ \\
\hline 4 & $-t^{3}+5 t^{2}-10 t+13-10 t^{-1}+5 t^{-2}-t^{-3}$ \\
\hline 5 & $t^{4}-6 t^{3}+15 t^{2}-24 t+29-24 t^{-1}+15 t^{-2}-6 t^{-3}+t^{-4}$ \\
\hline 10 & $-t^{9}+11 t^{8}-55 t^{7}+174 t^{6}-409 t^{5}+777 t^{4}-1243 t^{3}$ \\
& $+1716 t^{2}-2073 t+2207-2073 t^{-1}+1716 t^{-2}$ \\
& $-1243 t^{-3}+777 t^{-4}-409 t^{-5}+174 t^{-6}-55 t^{-7}+11 t^{-8}-t^{-9}$ \\
\hline 11 & $t^{10}-12 t^{9}+66 t^{8}-230 t^{7}+593 t^{6}-1232 t^{5}+2157 t^{4}-3268 t^{3}$ \\
& $+4356 t^{2}-5158 t+5455-5158 t^{-1}+4356 t^{-2}$ \\
& $-3268 t^{-3}+2157 t^{-4}-1232 t^{-5}+593 t^{-6}-230 t^{-7}+66 t^{-8}-12 t^{-9}+t^{-10}$ \\
\hline
\end{tabular}


Table 6: Jones polynomials for $W(3, n)$

\begin{tabular}{|c|c|}
\hline$n$ & $V_{W(3, n)}(t)$ \\
\hline 4 & $t^{4}-4 t^{3}+6 t^{2}-7 t+9-7 t^{-1}+6 t^{-2}-4 t^{-3}+t^{-4}$ \\
\hline 5 & $-t^{5}+5 t^{4}-10 t^{3}+15 t^{2}-19 t+21-19 t^{-1}+15 t^{-2}-10 t^{-3}+5 t^{-4}-t^{-5}$ \\
\hline 10 & $t^{10}-10 t^{9}+45 t^{8}-130 t^{7}+290 t^{6}-542 t^{5}+875 t^{4}$ \\
& $-1250 t^{3}+1600 t^{2}-1849 t+1941-1849 t^{-1}+1600 t^{-2}-1250 t^{-3}$ \\
& $+875 t^{-4}-542 t^{-5}+290 t^{-6}-130 t^{-7}+45 t^{-8}-10 t^{-9}+t^{-10}$ \\
\hline 11 & $-t^{11}+11 t^{10}-55 t^{9}+176 t^{8}-429 t^{7}+869 t^{6}-1518 t^{5}+2343 t^{4}$ \\
& $-3245 t^{3}+4070 t^{2}-4652 t+4863-4652 t^{-1}+4070 t^{-2}-3245 t^{-3}$ \\
& $+2343 t^{-4}-1518 t^{-5}+869 t^{-6}-429 t^{-7}+176 t^{-8}-55 t^{-9}+11 t^{-10}-t^{-11}$ \\
\hline
\end{tabular}

Table 7: HOMFLY-PT polynomials for $W(3, n)$

\begin{tabular}{|c|c|}
\hline$n$ & $H_{W(3, n)}(a, z)$ \\
\hline 4 & $a^{2}\left(z^{4}+z^{2}-1\right)+\left(-z^{6}-3 z^{4}-z^{2}+3\right)+a^{-2}\left(z^{4}+z^{2}-1\right)$ \\
\hline 5 & $a^{2}\left(-z^{6}-2 z^{4}+z^{2}+2\right)+\left(z^{8}+4 z^{6}+3 z^{4}-4 z^{2}-3\right)+a^{-2}\left(-z^{6}-2 z^{4}+z^{2}+2\right)$ \\
\hline 10 & $a^{2}\left(z^{16}+7 z^{14}+14 z^{12}-2 z^{10}-29 z^{8}-11 z^{6}+18 z^{4}+6 z^{2}-3\right)$ \\
& $+\left(-z^{18}-9 z^{16}-28 z^{14}-26 z^{12}+33 z^{10}+69 z^{8}+4 z^{6}-42 z^{4}-9 z^{2}+7\right)$ \\
& $+a^{-2}\left(z^{16}+7 z^{14}+14 z^{12}-2 z^{10}-29 z^{8}-11 z^{6}+18 z^{4}+6 z^{2}-3\right)$ \\
\hline 11 & $a^{2}\left(-z^{18}-8 z^{16}-20 z^{14}-6 z^{12}+40 z^{10}+34 z^{8}-25 z^{6}-24 z^{4}+6 z^{2}+4\right)$ \\
& $+\left(z^{20}+10 z^{18}+36 z^{16}+46 z^{14}-28 z^{12}-114 z^{10}-43 z^{8}+74 z^{6}+42 z^{4}-16 z^{2}-7\right)$ \\
& $+a^{-2}\left(-z^{18}-8 z^{16}-20 z^{14}-6 z^{12}+40 z^{10}+34 z^{8}-25 z^{6}-24 z^{4}+6 z^{2}+4\right)$ \\
\hline
\end{tabular}

\section{Notes on Computing}

This file contains some remarks on the roles played by Mathematica and Maple experiments in generating data, conjectures, and results.

Our initial interest was in the Khovanov homology of weaving knots, which we knew was determined in a straightforward manner by the Jones polynomials. It also turns out that the Khovanov homology of our knots can be determined by knowing half of the Khovanov homology, essentially. Instead of having to keep track of a bigraded object, the study of Khovanov homology of weaving knots is reduced to the study of a graded object. We normalized our examples by dividing each dimension in the graded object by the total dimension and plotted the results for a large number of the knots. In the plots bell-shaped curves appear as envelopes of the plots of the normalized dimensions. First, this led us to conjecture that the standard deviations of the bell curves may be an interesting invariant for the family of knots $W(3, n)$. 
As mentioned, the Jones polynomial of a weaving knot $W(3, n)$ determines the twovariable Khovanov polynomial of the bi-graded Khovanov homology. To simplify matters, we studied the Jones polynomial on its own terms. We knew that the Jones polynomials have the form

$$
V_{W(3, n)}(t)= \pm t^{-n}+\lambda_{-n+1} t^{-n+1}+\cdots \lambda_{n-1} t^{n-1}+ \pm t^{n}
$$

so we conjectured that $\lambda_{-n+k}=\lambda_{n-k}$ is a polynomial function of degree $k$ in $n$. The basis for this conjecture is the well-known binomial distribution approximating the standard normal distribution. To investigate this conjecture further, we detoured through another round of experiments.

During a visit to the University of Osnabrück in Germany, the second-named author was tutored in Mathematica by Prof. Dr. Karl-Heinz Spindler. During the demonstrations of techniques for manipulating polynomials, Dr. Spindler asked if we knew explanations of the patterns we were observing. These questions led to the formulation and proof of the palindromic properties of the building block polynomials $C_{n,-}(q)$ stated in theorem 3.7 .

For a large sample of computed Jones polynomials, we extracted the coefficients $\lambda_{-n+k}$ obtaining sequences of integers upon which Mathematica routines computed iterated differences. In accordance with the conjectured behavior, we observed the differences vanishing after the expected number of iterations. From these experiments it was possible to generate formulas for the numbers $\lambda_{-n+2}$ and $\lambda_{-n+3}$ eventually proved in theorems 5.1 and 5.2 .

One may also obtain expressions for two-variable HOMFLY-PT polynomial $H_{(W(3, n)}(a, z)$ normalized to $H$ (Unknot) $(a, z)=1$. This amounts to applying a different sequence of substitutions to the Hecke algebra output $V_{\left(\sigma_{1} \sigma_{2}^{-1}\right)^{n}}(q, z)$ given in (4.1). Since we have no immediate use for these gadgets, we offer the brief table 7.

Turning to the volume computations, we thank Ilya Kofman for a significant improvement of our first script for computing volumes using SnapPy [4]. According to our data, the volume of the complement of $W(3, n)$ is growing roughly linearly with $n$, so it is not so surprising that the volume is strongly correlated with the higher twist numbers. Another feature of the SnapPy data is that, although the volume is growing linearly, the number of simplices used by SnapPy to compute the volume is growing quite irregularly.

\section{References}

[1] Dror Bar-Natan. On Khovanov's categorification of the Jones polynomial. Algebr. Geom. Topol., 2:337-370, 2002.

[2] Joan S. Birman and William W. Menasco. A note on closed 3-braids. Commun. Contemp. Math., 10(suppl. 1):1033-1047, 2008.

[3] Abhijit Champanerkar, Ilya Kofman, and Jessica S. Purcell. Volume bounds for weaving knots. Algebr. Geom. Topol., 16(6):3301-3323, 2016.

[4] Marc Culler, Nathan M. Dunfield, Matthias Goerner, and Jeffrey R. Weeks. SnapPy, a computer program for studying the geometry and topology of 3-manifolds. Available at URL http://snappy.computop.org (01/10/2018). 
[5] Oliver T. Dasbach and Xiao-Song Lin. A volumish theorem for the Jones polynomial of alternating knots. Pacific J. Math., 231(2):279-291, 2007.

[6] Pierre de la Harpe, Michel Kervaire, and Claude Weber. On the Jones polynomial. Enseign. Math. (2), 32(3-4):271-335, 1986.

[7] Louis H. Kauffman. State models and the Jones polynomial. Topology, 26(3):395-407, 1987.

[8] Mikhail Khovanov. Patterns in knot cohomology. I. Experiment. Math., 12(3):365-374, 2003.

[9] Eun Soo Lee. An endomorphism of the Khovanov invariant. Adv. Math., 197(2):554-586, 2005.

[10] Ciprian Manolescu. An introduction to knot Floer homology. In Physics and mathematics of link homology, volume 680 of Contemp. Math., pages 99-135. Amer. Math. Soc., Providence, RI, 2016.

[11] Rama Mishra and Ross Staffeldt. On weaving knots. Available at http://wordpress.nmsu.edu/ross/on-weaving-knots, 2017.

[12] Rama Mishra and Ross Staffeldt. Distributions associated with weaving knots $W(3, n)$. In preparation, 2019.

[13] Peter Ozsváth and Zoltán Szabó. Heegaard Floer homology and alternating knots. Geom. Topol., 7:225-254, 2003.

[14] Peter Ozsváth and Zoltán Szabó. Holomorphic disks and genus bounds. Geom. Topol., 8:311-334, 2004.

[15] Jacob Rasmussen. Khovanov homology and the slice genus. Invent. Math., 182(2):419447, 2010.

[16] Dale Rolfsen. Knots and links, volume 346.H. AMS Chelsea, 2003.

[17] Alexander N. Shumakovitch. Torsion of the Khovanov homology. arXiv:math/0405474v2[math.GT], 19 June 2018.

[18] Moto-o Takahashi. Explicit formulas for Jones polynomials of closed 3-braids. Comment. Math. Univ. St. Paul., 38(2):129-167, 1989.

[19] William P. Thurston. Three-dimensional manifolds, Kleinian groups and hyperbolic geometry. Bull. Amer. Math. Soc. (N.S.), 6(3):357-381, 1982. 